\title{
Algebraic Discrete Nonlocal (DNL) Absorbing Boundary Condition for the Ship Wave Resistance Problem
}

\author{
M. Storti, J. D’Elía, and S. Idelsohn \\ Centro Internacional de Métodos Computacionales en Ingeniería (CIMEC), Instituto de Desarrollo \\ Tecnológico para la Industria Química (INTEC), Güemes 3450, 3000-Santa Fe, Argentina \\ E-mail: mstorti@minerva.unl.edu.ar
}

Received June 27, 1997; revised August 4, 1998; accepted August 7, 1998

\begin{abstract}
An absorbing boundary condition for the ship wave resistance problem is presented. In contrast to the Dawson-like methods, it avoids the use of numerical viscosities in the discretization, so that a centered scheme can be used for the free surface operator. The absorbing boundary condition is "completely absorbing," in the sense that the solution is independent of the position of the downstream boundary and is derived from straightforward analysis of the resulting constant-coefficients difference equations, assuming that the mesh is $1 \mathrm{D}$-structured (in the longitudinal direction) and requires the eigen-decomposition of a matrix one dimension lower than the system matrix. The use of a centered scheme for the free surface operator allows a full finite element discretization. The drag is computed by a momentum flux balance. This method is more accurate and guarantees positive resistances. (c) 1998 Academic Press
\end{abstract}

\section{INTRODUCTION}

When a body moves near the free surface of a fluid a pattern of trailing gravity waves is formed. The energy spent in building this pattern comes from the work done by the body against the wave resistance. Numerical modeling of this problem is a matter of high interest for ship design and marine engineering [1-12]. As a first approximation, the wave resistance can be computed with a potential model, whereas for the viscous drag it can be assumed that the position of the surface is held fixed at the reference hydrostatic position, i.e. a plane. This is, basically, the Froude hypotheses. With this assumption, we are neglecting the interaction produced by the boundary layer, which tends to produce a larger body, whose wave pattern, in turn, tends to modify the potential flow which is the input to the boundary layer process. Even if a potential model is assumed for the liquid, the problem is nonlinear due to the free surface boundary condition. 
We concentrate in this paper in the computation of the flow field and wave resistance for a body in steady motion, by means of a potential model for the fluid and a linearized free surface boundary condition. This is the basis for most ship design codes in industry. The governing equations are the Laplace equation with slip boundary conditions on the hull and channel walls, inlet/outlet conditions at the corresponding planes and the free surface boundary condition. The free surface boundary condition amounts to a Neumann boundary condition with a source term proportional to the streamlined second derivative of the potential. However, the problem as stated so far is ill posed, in the sense that it is invariant under longitudinal coordinate inversion $(x \rightarrow-x)$, and it is clear then, that it cannot capture the characteristic trailing waves propagating downstream. To do this, we can either add a dissipative numerical mechanism or impose some kind of "absorbing boundary condition."

It can be shown that the addition of a third-order derivative to the free surface boundary conditions, adds a dissipative mechanism and captures the correct sense of propagation for the wave pattern. This is equivalent to use a noncentered discretization scheme for the second-order operator and falls among the well-known "upwind-techniques." The amount of viscosity added is related to the length of the mesh downstream of the body. If the viscosity parameter is too low, the trailing waves arrive at the downstream boundary, are

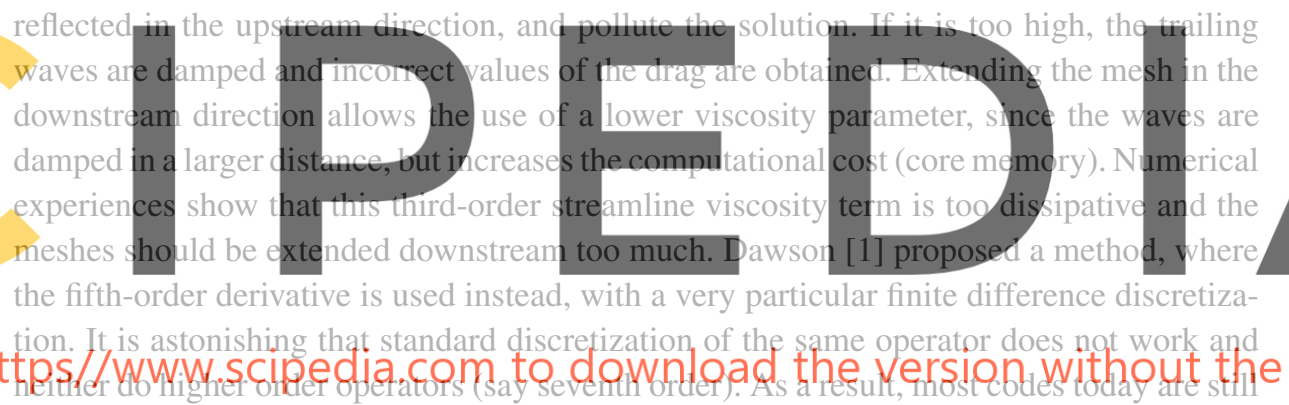
using some kind of variant of the Dawson scheme. However, this very particular viscosity term is hard to extend to general boundary fitted meshes, not to mention unstructured computational methods like finite elements. It is by this cause that most codes are based on a highly structured panel formulation.

Another possibility that is investigated in this work is to use an absorbing boundary condition in the downstream boundary. If such a numerical device could be found, then there is no need to add a numerical viscosity term, since the trailing waves are not reflected upstream, and a usual centered scheme can be used for the free surface boundary term. As a bonus, if such a centered scheme could be used, then the trailing waves would not dampen and the drag could be computed in terms of the momentum flow through a plane arbitrarily located downstream of the body. Absorbing boundary conditions are well studied for other wave phenomena [13-19] like the Helmholtz equation in acoustics but are harder to find in the context of the free surface flows studied in this paper. Broeze and Romate [2] developed an absorbing boundary condition for potential flow with a panel method but in the context of following a temporal evolution of the free surface problem and Lenoir and Tounsi [17] treated the "sea-keeping" problem, which is closer to the Helmholtz-like equation than the "wave-resistance" problem.

The absorbing boundary condition we develop here is based on straightforward study of the solutions of ODEs with constant coefficients on unbounded domains and follows closely the general case described in [15]. Once we solve for the roots of the characteristic equation, the unbounded domain solution downstream or upstream corresponds to retaining in the 
general solution, those terms that decay in that direction. For those roots with a null real part, a perturbation technique determines the sense of propagation. It can be shown that this is equivalent to determining the sign of the group velocity for the corresponding mode. Once the general unbounded domain solution is found, the absorbing boundary condition is obtained by differentiating this form and results in a full matrix coupling all the degrees of freedom on the outlet plane. This behavior is similar to the well-known DtN absorbing boundary condition proposed by Givoli and Keller in the context of the Helmholtz equation [13,14].

\section{GOVERNING EQUATIONS}

Consider the flow around a ship moving at constant speed and with fixed orientation in space (i.e. no pitching, yawing, or rolling are allowed) with respect to water at rest in a channel of constant section which, for simplicity, is assumed to be a rectangle of depth $H$ and width $L_{y}$ as shown in Fig. 1. We describe the flow in a system of coordinates attached to the ship, so that the ship is at rest and the flow and elevation pattern arrive at a steady pattern after an initial transient. The fluid to be modeled occupies region $\Omega$ which is bounded by the channel walls and bottom $\Sigma_{\mathrm{ch}}$, the inlet/outlet boundaries $\Sigma_{\text {in/out }}$, the wetted surface of the ship $\Sigma_{\mathrm{s}}$
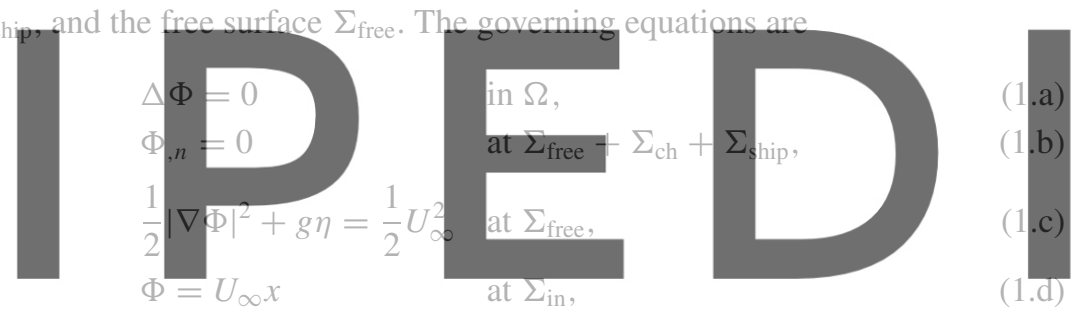

radiation b.c.'s

at $\Sigma_{\text {in }}$

thtps//www.scipedia.com to download the version without th The Laplace equation (1.a) comes from the assumption that the flow is irrotational and incompressible. The usual slip condition (1.b) is imposed at the channel walls, bottom, and free surface. Alternatively, we will consider also the case of imposing Dirichlet boundary conditions at the bottom: $\Phi=U_{\infty} x$. This is discussed both at the beginning of Section 4 and in Appendix 1. Equation (1.c) is the "dynamic free surface" boundary condition. It comes from the Bernoulli equation (including a hydrostatic term $g z, \eta$ stands for the surface elevation) and it is usually linearized under certain assumptions as, for instance, that the ship is thin, slender, slow, or deeply submerged. The "radiation boundary conditions" should

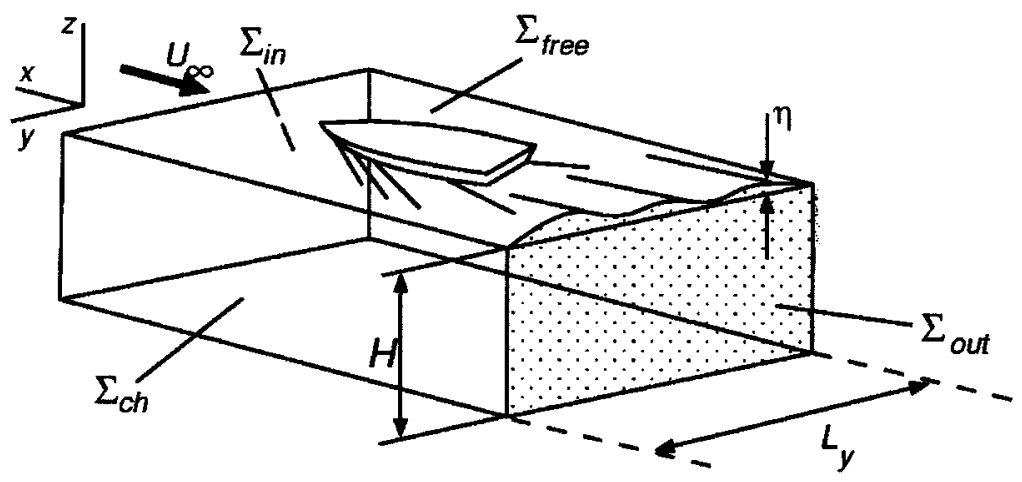

FIG. 1. Geometrical description. 
allow, roughly speaking, the flow of energy in the form of radiating waves to propagate downstream and exit cleanly at $\Sigma_{\text {out }}$. In contrast, no waves are allowed to propagate upstream to $\Sigma_{\text {in }}$ so that we simply impose that the potential should approach the undisturbed one there. Note that, the different treatment in $\Sigma_{\text {in }}$ and $\Sigma_{\text {out }}$ is the only element that can break the symmetry $x \rightarrow-x$ and ensure a physically correct wave pattern. Another means of doing this is the addition of some "upwind" or "numerical dissipation" mechanism.

In slow ship theory, the flow is decomposed in a base flow $\Phi_{0}$, also called "double body flow," and a "wave perturbation" flow $\phi[3,6,7]$ defined by

$$
\Phi=\Phi_{0}+\phi
$$

The great simplification comes from the fact that the governing equations for both flows are restricted to the domain $\Omega_{0}$, where the free surface $\Sigma_{\text {free }}$ has been replaced by the undisturbed position of the free surface $\Sigma_{\text {free } 0}$, which in this case is simply the plane $z=0$. The double body flow satisfies

$\Delta \Phi_{0}=0 \quad$ in $\Omega_{0}$,

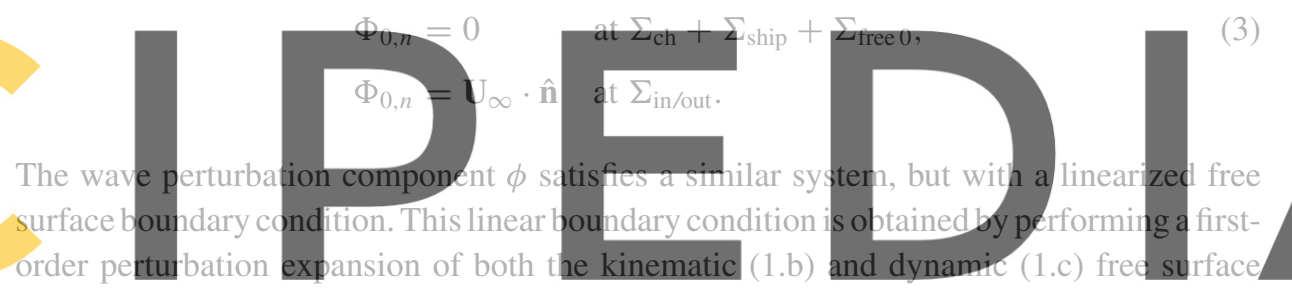
conditions and eliminating the surface elevation $\eta$. This results is a combined Neumann-

ht
tree surface. Several versions of the free surface boundary condition have been proposed (see [1-12], especially [7-9]), which depend on the particular assumption (i.e. slow ship, thin ship, or deeply submerged ship, for instance). We will make use here of a rather standard slow ship expansion, but the process of developing the absorbing boundary condition is rather independent of the specific linearized free boundary condition used. The governing equations for the wave perturbation potential are

$$
\begin{array}{ll}
\Delta \phi=0, & \text { in } \Omega_{0}, \\
\phi_{, n}=0, & \text { at } \Sigma_{\mathrm{ch}}+\Sigma_{\mathrm{ship}}, \\
\phi=0, & \text { at } \Sigma_{\mathrm{in}}, \\
\phi_{, n}+\frac{\partial}{\partial x_{i}}\left(U_{0 i} U_{0 j} \phi, j\right)=\text { r.h.s., } & \text { at } \Sigma_{\mathrm{free}}, \\
\text { radiation b.c.'s, } & \text { at } \Sigma_{\mathrm{out}} .
\end{array}
$$

Here $U_{0 i}=\Phi_{0, i}$ and r.h.s. is a right-hand side term involving the residual of the Bernoulli equation at the reference free surface $z=0$ for the base flow. This instance of the boundary condition has the advantage that it can be easily treated in a finite element context, since the conservative form of the streamlined second-order derivative can be integrated by parts. However, it will be clear from the development through the paper that the proposed boundary condition can be easily coupled to any version of the linearized free surface boundary conditions. It will also be explained for a finite element (FEM) discretization, but it is automatically extended to any other kind of "in volume" methods: finite volumes (FVM), 
finite differences (FDM) or the younger "mesh-less methods" [20,21]. The extension to boundary integral methods as the "panel" or "boundary element" ones is still in development.

We will describe the method in a more restricted context, by replacing the ship by a pressure distribution, i.e. nonuniform atmospheric pressure. Physically, this problem corresponds to hovercrafts, and the nonlinear free surface boundary condition is

$$
P(\mathbf{x})+\frac{1}{2} \rho|\nabla \Phi|^{2}+\rho g \eta=P_{\mathrm{atm}}+\frac{1}{2} \rho U_{\infty}^{2}
$$

where $P_{\text {atm }}$ is the atmospheric pressure far from the disturbance and $P(\mathbf{x})$ is a given function. It is clear that once the absorbing boundary condition is obtained, it can be applied to the more general ship case, defined by (4), since the absorbing properties are independent of the generating mechanism. The 3D examples below correspond to the general case of Eqs. (4). Assuming that the disturbance is small, i.e. $\left|P-P_{\text {atm }}\right| \ll \frac{1}{2} \rho U_{\infty}^{2}$, we can take $\Phi_{0}=U_{\infty} x$ as the base flow and we obtain the following linear problem for the wave perturbation potential:

$$
\begin{array}{ll}
\Delta \phi=0, & \text { in } \Omega_{0}, \\
\phi_{, n}=0, & \text { at } \Sigma_{\mathrm{ch}},
\end{array}
$$

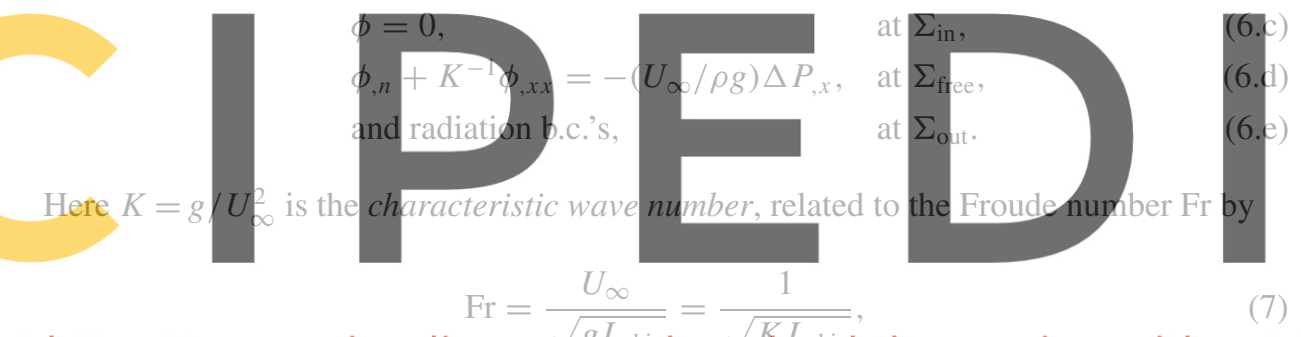

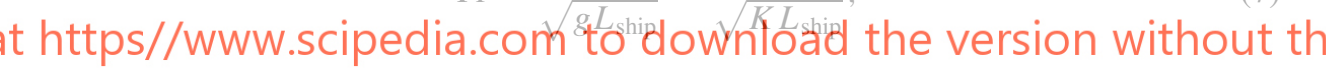

where $L_{\text {ship }}$ is a characteristic length of the ship. This problem is far more simple than the previous one since the involved operator plus boundary conditions are invariant under a translation in the $x$ direction. In addition, we will assume that the pressure perturbation has compact support, i.e., that

$$
\Delta P \equiv 0 \text { for }|x|>L_{\Delta P}
$$

\section{PARTIAL DISCRETIZATION}

To apply the method, we have first to discretize the problem in order to obtain a system of ODEs. Let $\Sigma_{y z}$ be the typical section of the channel (see Fig. 2); then we will discretize the problem in two steps by doing, first, a partial discretization in $\Sigma_{y z}$ and, then, a onedimensional discretization in the $x$ coordinate. We will show how the partial discretization by finite elements is performed; the case of finite differences is straightforward. Having a two-dimensional finite element mesh in the typical section we approximate the potential by

$$
\phi(x, y, z) \sim \hat{\phi}(x, y, z)=\sum_{k=1}^{N_{\text {slab }}} \hat{\phi}_{k}(x) N_{k}(y, z),
$$

where $N_{\text {slab }}$ is the number of "free" nodes in the typical section; i.e., they do not include the bottom nodes if Dirichlet boundary conditions are imposed in that part of the boundary. 


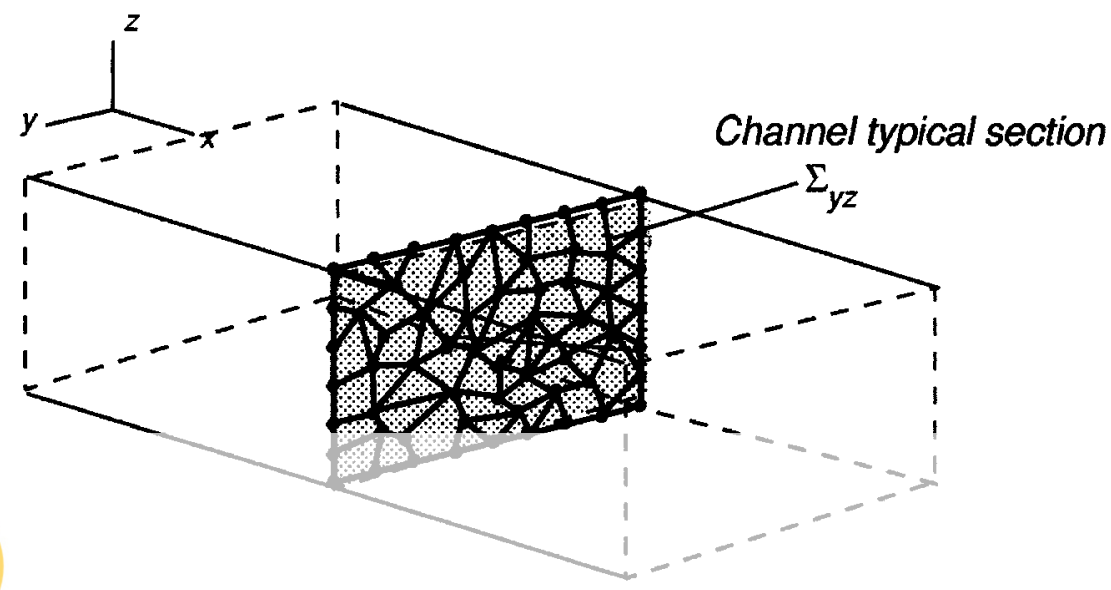

FIG. 2. Discretization of the problem as the composition of a 2D discretization in $y z$ and a $1 \mathrm{D}$ discretization in $x$. FEM unstructured mesh on the typical channel section.

$N_{k}(y, z)$ are two-dimensional interpolation functions. Replacing this in the Laplace equation (6.a) and integrating by parts in $y z$, we obtain where $\phi(x)$ is the vector of nodal potentials and $\mathbf{M} \mathbf{I}$ and $\mathbf{K}$ a
the identity (mass matrix) and Laplace operators, defined by

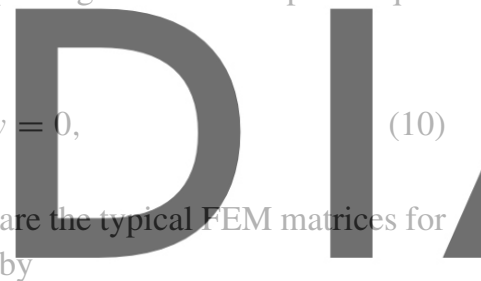

https//www.scipedia.con $\hat{h}_{1}$ to download the version without the

$$
\begin{aligned}
\phi(x) & =\left[\begin{array}{c}
\hat{\phi}_{2}(x) \\
\vdots \\
\hat{\phi}_{N_{\text {slab }}}(x)
\end{array}\right], \\
M_{j k} & =\int_{\Sigma_{y z}} N_{j}(y, z) N_{k}(y, z) d y d z, \\
K_{j k} & =\int_{\Sigma_{y z}} \nabla_{y z} N_{j}(y, z) \cdot \nabla_{y z} N_{k}(y, z) d y d z .
\end{aligned}
$$

Replacing $\phi_{, n}$ from the free surface boundary condition (6.d), we arrive at

$$
\tilde{\mathbf{M}} \phi_{, x x}-\mathbf{K} \phi=\mathbf{G}(x),
$$

which is a system of ODEs. The modified mass matrix $\tilde{\mathbf{M}}$ includes the "free surface mass matrix" $\mathbf{M}_{\text {free }}$, and $\mathbf{G}(x)$ is a right-hand side contribution coming from the pressure perturbation on the free surface, defined by

$$
\tilde{\mathbf{M}}=\mathbf{M}-K^{-1} \mathbf{M}_{\text {free }},
$$

where

$$
M_{\text {free }, j k}=\int_{\Sigma_{\text {free } 0}} N_{j}(y, 0) N_{k}(y, 0) d y
$$


and

$$
G_{k}(x)=\left(U_{\infty} / \rho g\right) \int_{\Sigma_{\text {free } 0}} N_{k} \Delta P_{, x} d y .
$$

$\mathbf{M}$ and $\mathbf{M}_{\text {free }}$ are positive definite and semi-definite mass matrices, respectively, $\mathbf{K}$ is positive semi-definite if Neumann boundary conditions are imposed at the bottom, and positive definite if Dirichlet boundary conditions are imposed at the bottom. All of them are symmetric. Due to the negative sign in (13) $\tilde{\mathbf{M}}$ does not have a definite sign.

\section{MODAL DECOMPOSITION}

The general solution to (12) can be found by means of a modal decomposition. Let $\mathbf{S}, \Lambda$ be the solution to the eigenvalue decomposition problem,

$$
\mathbf{K S}=\tilde{\mathbf{M S}} \Lambda
$$

with S nonsingular and $\Lambda$ diagonal. For a Dirichlet boundary condition at the bottom, this decomposition is assured by the fact that both $\tilde{\mathbf{V i}}$ and $\mathbf{K}$ are symmetricand real, and $\mathbb{K}$
is positive definite. For a Neumann boundary condition at the bottom $\mathbf{K}$ is only positive
semi-definite, but the null eigenyector of the matrix $\mathbb{K}$ can be treated separately and the
problem is reduced again to $\mathbf{K}$ positive definite (for details, refer to Appendix 1 ). We can
assume also that $\mathrm{S}$ and $\boldsymbol{\Lambda}$ are real. We make the change of variables $\phi=\mathbf{S U}$, and system
(12) becomes, after premultiplying by $\mathrm{S}^{-1} \tilde{\mathbf{M}}^{-1}$, a set of uncoupled equations of the form

at https//www.scipedia.com to downlorad the version without th where

$$
\mathbf{F}(x)=\mathbf{S}^{-1} \tilde{\mathbf{M}}^{-1} \mathbf{G}(x) .
$$

For small Froude numbers (large $K$ ): (i) the free surface term can be neglected in (13), so that the problem is equivalent to the Laplace equation with some prescribed flux on the free surface; and (ii) the matrix $\tilde{\mathbf{M}}=\mathbf{M}$ is positive definite and all the eigenvalues $\left\{\lambda_{k}\right\}$ are positive, corresponding to the purely viscous case. But, for the Froude numbers of interest (typically $\operatorname{Fr} \gtrsim\left(\Delta z / 3 L_{\text {ship }}\right)^{1 / 2}$, where $\Delta z$ is the typical vertical size of the elements near the surface; see Appendix 2), $N_{\text {inv }}$ eigenvalues become negative, and we may assume that

$$
\begin{array}{ll}
\lambda_{k}<0 & \text { for } 1 \leq k \leq N_{\mathrm{inv}}, \\
\lambda_{k}>0 & \text { for } N_{\mathrm{inv}}+1 \leq k \leq N_{\text {slab }} .
\end{array}
$$

Each negative $\lambda_{k}$ gives two complex conjugate inviscid $\mu$ eigenvalues of the form $\mu_{k}^{ \pm}=$ $\pm i \sqrt{\left|\lambda_{k}\right|}$ (see Fig. 3).

The key part of the method relies on the solution of each of the $1 \mathrm{D}$ equations (17). Physically, a radiation boundary condition is such that when it is imposed on a boundary it gives the same solution as if the boundary were "pushed" to infinity, i.e. the "unbounded domain solution." We will see that for the positive $\lambda_{k}$ 's, this process of pushing the boundary to infinity has a well-defined limit, whereas for the negative $\lambda_{k}$ 's, a perturbation analysis 


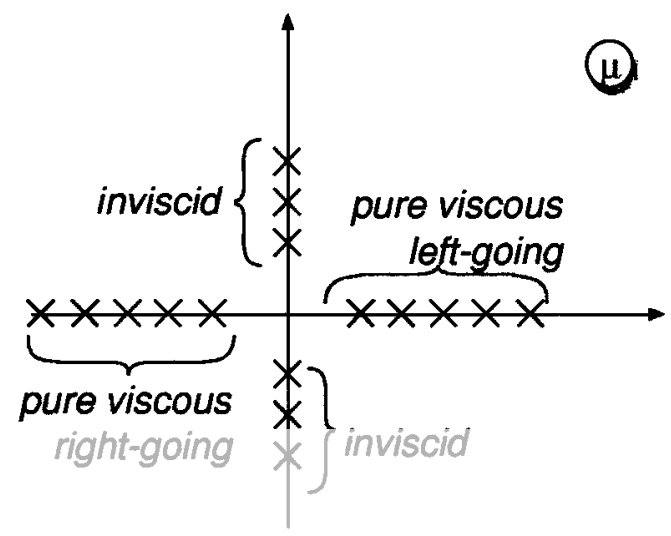

FIG. 3. Eigenvalue distribution for the inviscid free surface problem.

will be required. The treatment given here resembles the one given by Hagstrom and Keller [15], but here we consider in detail the case of these negative eigenvalues (i.e., not exhibiting a naturalexponential decay or growth)

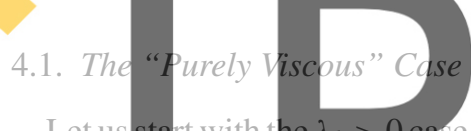

Let us start with the $\lambda_{k}>0$ case, which is equivalent to a 10 cooling in $|x|<L$ and homogeneous Di we will drop the $k$ subindex and, as we
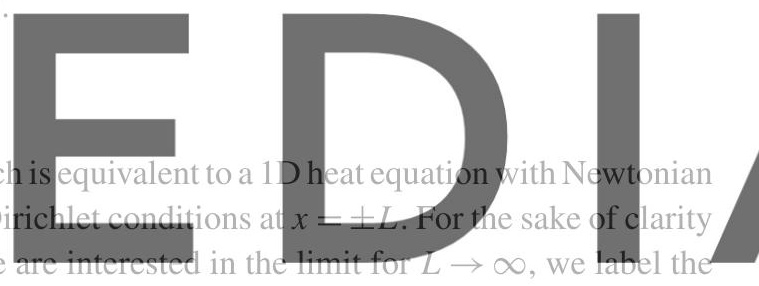
corresponding solution as $U_{L}$, which satisfies

https//www.scipedia.com to download the version without the

$$
\begin{array}{ll}
U_{L, x x}-\lambda U_{L}=F(x) & \text { in }|x|<L, \\
U_{L}=0 & \text { at } x= \pm L,
\end{array}
$$

with $\lambda>0$. Due to the compact support of $\Delta P$ assumed in (8), $F$ has the same compact support, i.e.

$$
F(x)=0 \text { for }|x|>L_{\Delta P}
$$

The solution to this is of the form

$$
U_{L}=U_{L, \mathrm{NH}}+U_{L, \mathrm{H}},
$$

where $U_{L, \mathrm{NH}}$ is a nonhomogeneous solution satisfying (20.a) but not necessarily the boundary conditions, and $U_{L, \mathrm{H}}$ is the general homogeneous solution, found by looking for solutions of the form $e^{\mu x}$ and solving the characteristic polynomial which in this case leads to $\mu= \pm k$ with $k=\sqrt{\lambda}$ (see Fig. 4). Then, the general homogeneous solution is

$$
U_{L, \mathrm{H}}(x)=a e^{k x}+b e^{-k x}
$$

But, due to the assumption of compact support for the pressure perturbation (8), $F$ is null for $|x|>L_{\Delta P}$ and, then, an expression of the form (23) holds for $U_{L, \mathrm{NH}}$ in the region $x>L_{\Delta P}$ 


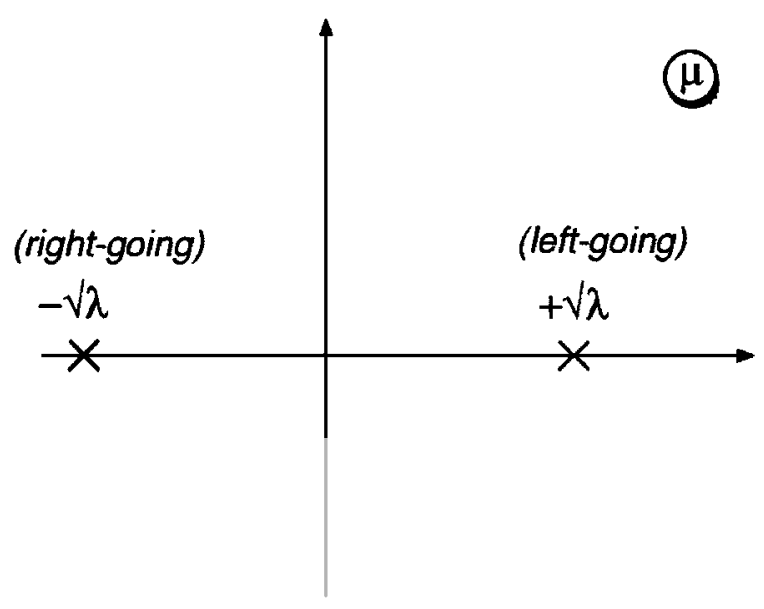

FIG. 4. Pure viscous case (heat equation with Newtonian cooling). The $\mu$ eigenvalues are on the real axis. and $x<-L_{\Delta P}$. However, the coefficients are different for both regions,
The constants $c$ and $b$
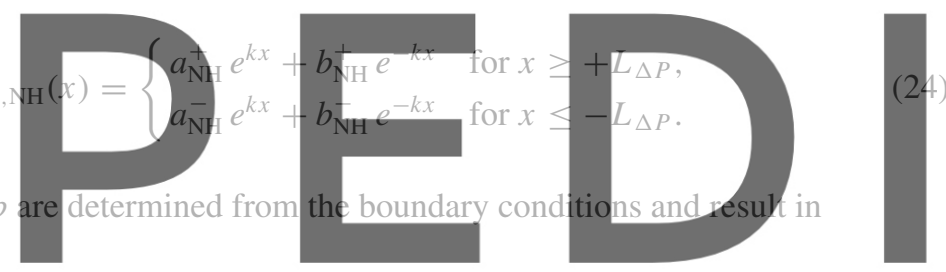
at https//www.scipedia.com to download the
\[ b=\frac{-b_{\mathrm{NH}}^{-} e^{2 k L}+b_{\mathrm{NH}}^{+} e^{-2 k L}+b_{\mathrm{NH}}^{+}-b_{\mathrm{NH}}^{-}}{e^{2 k L}-e^{-2 k L}} \]

and, letting $L \rightarrow \infty$, we obtain

$$
\lim _{L \rightarrow \infty} a=-a_{\mathrm{NH}}^{+}, \quad \lim _{L \rightarrow \infty} b=-b_{\mathrm{NH}}^{-} .
$$

This defines the "unbounded domain solution" $\lim _{L \rightarrow \infty} U_{L}$ for the problem. It is clear that it is unique from the unicity of the bounded solution. It can also be shown that it is independent of the kind of boundary condition imposed at the artificial boundaries $x= \pm L$. Moreover, it satisfies

$$
\lim _{L \rightarrow \infty} U_{L}= \begin{cases}\left(b_{\mathrm{NH}}^{+}-b_{\mathrm{NH}}^{-}\right) e^{-k x} & \text { for } x>+L_{\Delta P}, \\ \left(a_{\mathrm{NH}}^{-}-a_{\mathrm{NH}}^{+}\right) e^{+k x} & \text { for } x<-L_{\Delta P} .\end{cases}
$$

So that the "unbounded domain solution" is such that it has only decaying components for $x \rightarrow+\infty$ in $x>L_{\Delta P}$ and vice versa for the left boundary. If we classify a decaying component $+\infty$, such as $e^{-k x}$, as "right-going," and $e^{k x}$ as "left going," then we recover the well-known rule for imposing boundary conditions in the context of hyperbolic systems: impose the incoming components to zero and let free the outgoing components. This suggests the following modification of (20), including "absorbing" boundary conditions based on 
differentiation of the forms in (27) as

$$
\begin{aligned}
U_{, x x}-\lambda U=F(x) & \text { in }|x|<L \\
U_{, x}+k U=0 & \text { at } x=L>L_{\Delta P} \\
U_{, x}-k U=0 & \text { at } x=-L<-L_{\Delta P} .
\end{aligned}
$$

It can be shown that the solution to this system agrees with $\lim _{L \rightarrow \infty} U_{L}$, independently of $L$, as long as $L>L_{\Delta P}$, of course. Equations (28.b), (28.c) are then referred to as "completely absorbing boundary conditions."

\subsection{The Inviscid Case}

Now consider (20) in the $\lambda_{k}<0$ case, which is a $1 \mathrm{D}$ Helmholtz equation. The characteristic equation leads now to $\mu= \pm i k$ (see Fig. 5) with $k=\sqrt{|\lambda|}$ and the general solution to the homogeneous equation is now of the form $a e^{i k x}+b e^{-i k x}$. After imposing the boundary conditions, we obtain

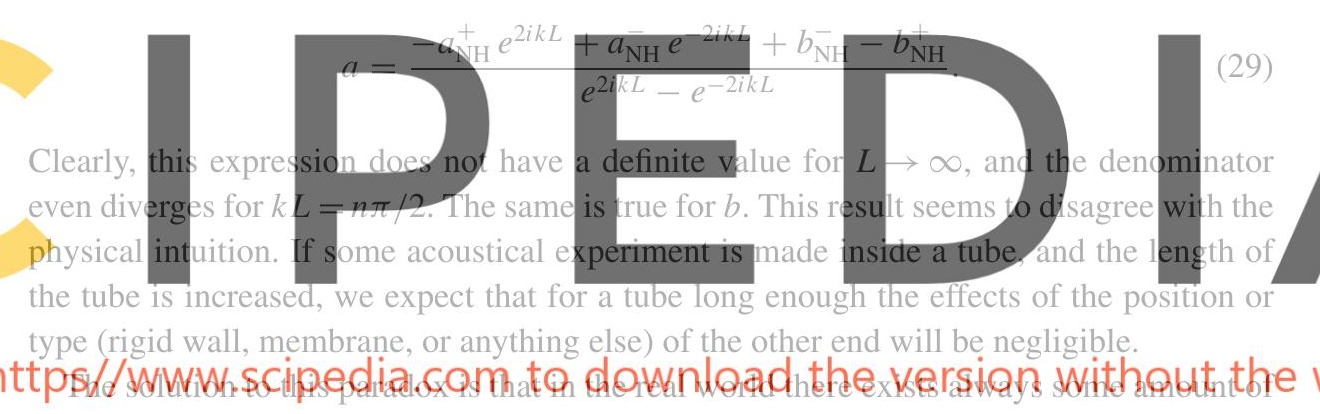

physical dissipation. Roughly speaking, we propose to add a dissipation term, classify the modes as right- or left-going and then let the dissipation parameter go to zero. However, the resulting absorbing boundary conditions will depend on the particular dissipation operator chosen, and then the conclusion is that the dissipation operator has to be chosen as close to the physics of the problem at hand as possible, such that the sense of propagation of the

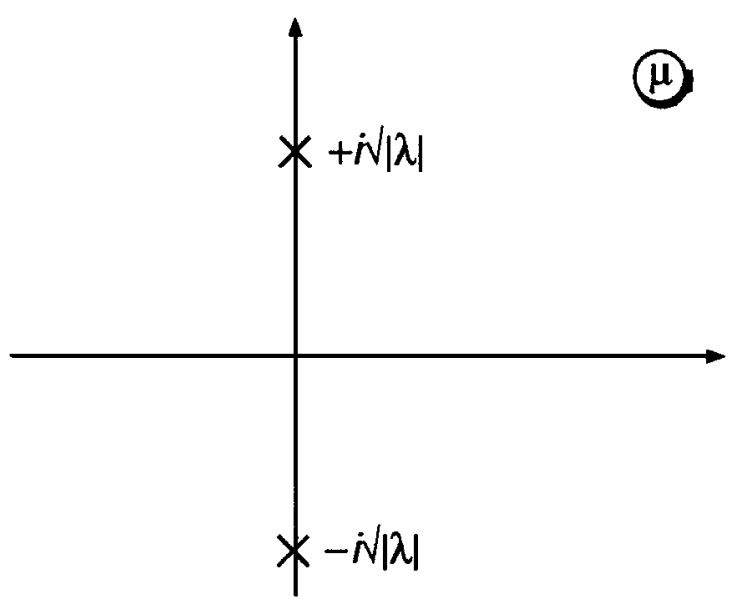

FIG. 5. Inviscid case (Helmholtz equation). The $\mu$ eigenvalues are on the imaginary axis. 
undamped waves is preserved. As an example, we will first derive the absorbing boundary conditions for the Helmholtz equation, as coming from the wave equation in the frequency domain. Later, we will derive the absorbing boundary conditions for the inviscid modes, $\lambda_{k}<0$. Even if it is equivalent to a Helmholtz equation, it will be shown that the physical dissipation appropriated for the wave-resistance problem leads to an absorbing boundary condition that is essentially different from the wave equation in the frequency domain.

4.2.1. The 1D Helmholtz equation. Consider the 1D Helmholtz equation with homogeneous Dirichlet boundary conditions at $x= \pm L$,

$$
\begin{array}{ll}
u_{\delta L, x x}+\left(\kappa^{2}+i \delta\right) u_{\delta L}=F & \text { in }|x|<L, \\
u_{\delta L}=0 & \text { at } x= \pm L
\end{array}
$$

where $u_{\delta L}$ is the amplitude of the perturbation, $\omega=c \kappa$ is the dispersion law, with $\omega$ the frequency and $c$ the speed of sound. Dissipation has been introduced by adding a term $i \delta u$, with $\delta>0, \delta \rightarrow 0$. The characteristic eigenvalues are $\mu= \pm i k_{\delta}$ (see Fig. 6), where $k_{\delta}$ is the complex solution of

$$
k_{\delta}^{2}=\kappa^{2}+i \delta
$$

with $\operatorname{Re}\left\{k_{\delta}\right\}>0$. As $\kappa^{2}+i \delta$ is in the first quadrant, $k_{\delta}$ will be there also, i.e. $\operatorname{Im}\left\{k_{\delta}\right\}>0$, and then

$$
\left|e^{ \pm i k_{\delta} x}\right| \rightarrow \begin{cases}\infty & \text { for } x \rightarrow \mp \infty \\ 0 & \text { for } x \rightarrow \pm \infty\end{cases}
$$

so that $i k_{\delta}$ is right-going and $-i k_{\delta}$ is left-going. The general expression for the homogeneous solution is of the form

$$
u_{\mathrm{H}}=a e^{-i k_{\delta} x}+b e^{i k_{\delta} x}
$$

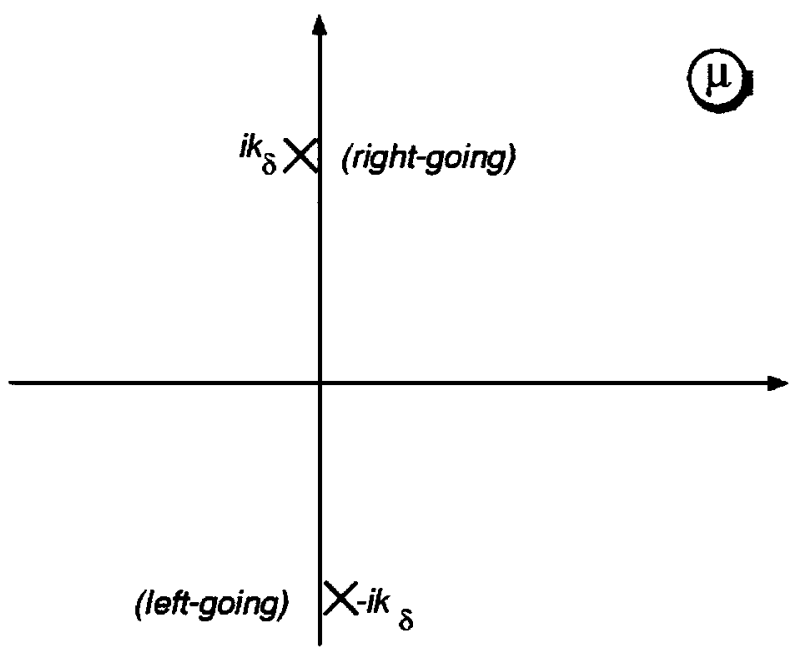

FIG. 6. The inviscid eigenvalues are "perturbed" with a small dissipative term. 
Forming the general solution and solving for the constants,

$$
\begin{aligned}
& a=\frac{-a_{\mathrm{NH}}^{+} e^{-2 i k_{\delta} L}+a_{\mathrm{NH}}^{-} e^{2 i k_{\delta} L}+b_{\mathrm{NH}}^{-}-b_{\mathrm{NH}}^{+}}{e^{-2 i k_{\delta} L}-e^{2 i k_{\delta} L}}, \\
& b=\frac{-b_{\mathrm{NH}}^{-} e^{-2 i k_{\delta} L}+b_{\mathrm{NH}}^{+} e^{2 i k_{\delta} L}+b_{\mathrm{NH}}^{+}-b_{\mathrm{NH}}^{-}}{e^{-2 i k_{\delta} L}-e^{2 i k_{\delta} L}},
\end{aligned}
$$

and again,

$$
\lim _{L \rightarrow \infty} a=-a_{\mathrm{NH}}^{+}, \quad \lim _{L \rightarrow \infty} b=-b_{\mathrm{NH}}^{-}
$$

As in (27), only the decaying components are retained in the far-field expansion, so that

$$
\lim _{L \rightarrow \infty} u_{\delta L}(x) \propto \begin{cases}e^{+i k_{\delta} x} & \text { for } x>+L_{\Delta P}, \\ e^{-i k_{\delta} x} & \text { for } x<-L_{\Delta P}\end{cases}
$$

This is the far-field expansion for the "viscous unbounded domain" solution for the problem, and it can be shown that it does not depend on the particular boundary condition imposed at $x=L$. The "inviscid unbounded domain" is obtained by letting $\delta \rightarrow 0$ and is simply

$$
u(x)=\lim _{\delta \rightarrow 0}\left\{\lim _{L \rightarrow \infty} u_{\delta L}(x)\right\}
$$

This is the solution we want. A key point is that the order in taking the limits does matter.

As $k_{\delta} \rightarrow k$ for $\delta \rightarrow 0$ it results that $u \propto e^{i k x}$ for $x>L_{\Delta P}$, and then

$$
u_{, x}-i k u=0 \quad \text { at } x=+L>L_{\Delta P}
$$

is the appropriate right boundary condition, whereas

$$
u_{, x}+i k u=0 \quad \text { at } x=-L<-L_{\Delta P}
$$

is the appropriate left one. Again, the solution to the Helmholtz 1D equation with these boundaries is the same as the limit solution (37), independently of $L>L_{\Delta P}$. It can be shown that the definition of right- and left-going are physically correct. Indeed, coming back to the time domain, it results that right-going waves do have a positive $+c$ group velocity, whereas the left-going ones have a negative $-c$ group velocity.

4.2.2. The wave-resistance problem. Other numerical algorithms, notably those based on the work of Dawson, do not use radiation boundary conditions, but instead they add a numerical viscosity term proportional to $\delta \phi_{, x x x x x}$, where $\delta$ is a "numerical viscosity parameter" or, also, a "Rayleigh viscosity coefficient." A term proportional to $-\delta \phi_{, x x x}$ is also dissipative, but the damping of the waves is too strong to be admissible for numerical calculations. Since after determination of the sense of propagation we take the limit $\delta \rightarrow 0$, precision does not matter here, and we choose by simplicity the low order $\phi_{, x x x}$ dissipative term. The perturbed free surface boundary condition is

$$
\phi_{, n}+K^{-1}\left(\phi_{, x x}-\delta \phi_{, x x x}\right)=-\left(U_{\infty} / \rho g\right) \Delta P_{, x}
$$


Repeating the semi-discretization process we arrive at the perturbed system of ODEs,

$$
\mathbf{M} \phi_{, x x}-K^{-1} \mathbf{M}_{\text {free }}\left(\phi_{, x x}-\delta \phi_{, x x x}\right)-\mathbf{K} \phi=\mathbf{G}(x),
$$

and the characteristic equation is obtained by replacing $\phi=\phi_{\delta} e^{\mu_{\delta} x}$ and results in

$$
\left(\mu_{\delta}^{2} \tilde{\mathbf{M}}+\delta K^{-1} \mu_{\delta}^{3} \mathbf{M}_{\text {free }}-\mathbf{K}\right) \phi_{\delta}=0 .
$$

To find the sense of propagation it is not necessary to determine the whole dependency of $\mu$ on $\delta$ but only a first-order expansion about $\delta=0$. Accordingly, we assume a regular perturbation expansion for $\mu_{\delta}$ and $\phi_{\delta}$ of the form

$$
\begin{aligned}
& \mu_{\delta}=\mu+\Delta \mu+O\left(\delta^{2}\right), \\
& \phi_{\delta}=\phi_{0}+\Delta \phi+O\left(\delta^{2}\right),
\end{aligned}
$$

where $\mu^{2}$ and $\phi_{0}$ are the eigenvalues and eigenvectors of the unperturbed system (16), i.e. the diagonal elements of $\boldsymbol{\Lambda}$ and column vectors of $\mathbf{S}$, respectively. Replacing Eqs. (43) in (42) and retaining only those terms up to $O(\delta)$, we obtain

$$
\left[\left(\mu^{2} \tilde{\mathbf{M}}-\mathbf{K}\right)+2 \mu \tilde{\mathbf{M}} \Delta \mu+\mu^{3} K^{-1} \mathbf{M}_{\text {free }} \delta\right]\left(\phi_{0}+\Delta \phi\right)=0 .
$$

Premultiplying by $\phi_{0}^{\mathrm{T}}$, we obtain

$$
\Delta \mu=-\frac{1}{2} \delta \mu^{2} K^{-1} \frac{\phi_{0}^{\mathrm{T}} \cdot \mathbf{M}_{\mathrm{free}} \cdot \phi_{0}}{\phi_{0}^{\mathrm{T}} \cdot \tilde{\mathbf{M}} \cdot \phi_{0}} .
$$

It follows from (16) that

$$
\phi_{0}^{\mathrm{T}} \cdot \mathbf{K} \cdot \phi_{0}=\mu^{2} \phi_{0}^{\mathrm{T}} \cdot \tilde{\mathbf{M}} \cdot \phi_{0}
$$

so that

$$
\Delta \mu=-1 / 2 \delta \mu^{4} K^{-1} \frac{\phi_{0}^{\mathrm{T}} \cdot \mathbf{M}_{\mathrm{free}} \cdot \phi_{0}}{\phi_{0}^{\mathrm{T}} \cdot \mathbf{K} \cdot \phi_{0}} .
$$

But, from (13) and (16), we have that for the inviscid modes

$$
|\lambda| K^{-1} \phi_{0}^{\mathrm{T}} \mathbf{M}_{\text {free }} \phi_{0}=\phi_{0}^{\mathrm{T}} \mathbf{K} \phi_{0}+|\lambda| \phi_{0}^{\mathrm{T}} \mathbf{M} \phi_{0}>0
$$

and, since $\mu$ is pure imaginary for the inviscid modes $\mu^{4}>0$, then $\Delta \mu$ is negative, so that all the inviscid eigenvalues are right-going (see Fig. 7).

It can be shown in a more general context that the "sense of propagation" as defined here has a correct physical sense, since "right going" modes correspond to positive group velocities for the time-dependent problem. Indeed, it can be shown that

$$
\Delta \mu=-\frac{\alpha \delta}{v_{G x}},
$$

where $v_{G x}$ is the $x$-component of the group velocity, and $\alpha$ is a real positive constant. 


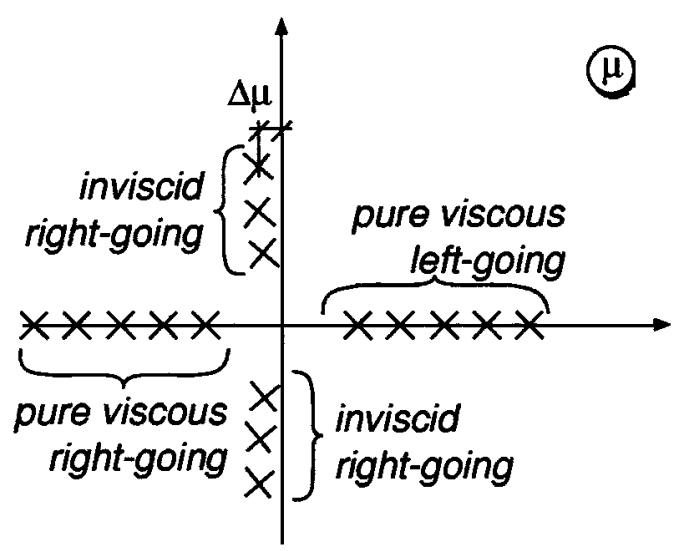

FIG. 7. Distributions of $\mu$ eigenvalues for the free surface problem with a small dissipative term.

As for each $\lambda_{k}<0$ both eigenvalues $\mu= \pm i \sqrt{\left|\lambda_{k}\right|}$ are right-going, the unbounded domain solution is

$$
U_{k}= \begin{cases}a_{k} e^{+i \sqrt{\left|\lambda_{k}\right|}}+b_{k} e^{-i \sqrt{\left|\lambda_{k}\right|} x} & \text { for } x>L_{\Delta P}, \\ 0 & \text { for } x<-L_{\Delta P},\end{cases}
$$

for $1 \leq k \leq N_{\text {inv }}$, where $x>L_{\Delta P}$ is far downstream and $x<L_{\Delta P}$ is far upstream. The corresponding radiation boundary conditions are

$$
\begin{array}{ll}
U_{k}=U_{k, x}=0 & \text { at } x=-L<-L_{\Delta P}, \\
\text { none } & \text { at } x=L>L_{\Delta P} .
\end{array}
$$

\subsection{Summary of Cases}

In brief, the method can be described as follows:

- Look for solutions with a dependency $\propto e^{\mu x}$ in the $x$ direction

- Solve the characteristic equation for the eigenvalues $\{\mu\}$. They are classified according to:

$\triangleright$ Viscous if $\operatorname{Re}\{\mu\} \neq 0$; inviscid otherwise.

$\triangleright$ The viscous modes are classified as right-going, if $\operatorname{Re}\{\mu\}<0$; left-going if $\operatorname{Re}\{\mu\}>0$.

$\triangleright$ To classify the inviscid modes, add a small dissipation and classify them as in the previous point.

- Retain only the right-going modes (viscous or inviscid) in the general expression.

- The radiation boundary condition is found by differentiation of this general form.

Of course, this corresponds to a boundary which is located at the right end of the domain. For a boundary at the left end, the left-going modes should be retained.

\section{THE RADIATION BOUNDARY CONDITION}

The absorbing boundary conditions for the viscous modes downstream (28.b) (see Fig. 8) can be written as

$$
\boldsymbol{\Pi}_{\mathrm{visc} U}\left(\mathbf{U}_{, x}+\boldsymbol{\Lambda}^{\frac{1}{2}} \mathbf{U}\right)=\mathbf{0}
$$




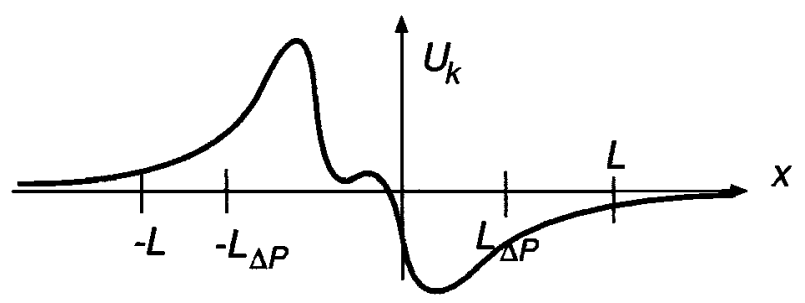

FIG. 8. Mode amplitude for the pure viscous modes.

where

$$
\begin{aligned}
\boldsymbol{\Lambda}^{\frac{1}{2}} & =\operatorname{diag}\{\underbrace{0, \ldots, 0}_{N_{\text {inv }}}, \sqrt{\lambda_{N_{\text {inv }}+1}}, \ldots, \sqrt{\lambda_{N_{\text {slab }}}}\}, \\
\boldsymbol{\Pi}_{\text {visc } U} & =\left[\mathbf{0}_{N_{\text {visc }} \times N_{\text {inv }}} \quad \mathbf{I}_{N_{\text {visc }} \times N_{\text {visc }}}\right] .
\end{aligned}
$$

$\boldsymbol{\Pi}_{\mathrm{visc} U}$ is the projection matrix on the subspace spanned by the viscous modes in the $\mathbf{U}$ basis. Coming back to the $\phi$ basis, we obtain

$$
\boldsymbol{\Pi}_{\mathrm{visc}}\left(\phi_{, x}+\mathbf{F}_{\mathrm{abs}} \phi\right)=\mathbf{0} \quad \text { at } x=+L,
$$

where

$$
\begin{aligned}
\boldsymbol{\Pi}_{\mathrm{visc}} & =\boldsymbol{\Pi}_{\mathrm{visc} U} \mathbf{S}^{-1}, \\
\mathbf{F}_{\mathrm{abs}} & =\mathbf{S} \boldsymbol{\Lambda}^{\frac{1}{2}} \mathbf{S}^{-1},
\end{aligned}
$$

and, similarly, upstream,

$$
\boldsymbol{\Pi}_{\mathrm{visc}}\left(\phi, x-\mathbf{F}_{\mathrm{abs}} \phi\right)=\mathbf{0} \quad \text { at } x=-L .
$$

$\boldsymbol{\Pi}_{\mathrm{visc}}$ is the same projection operator as $\boldsymbol{\Pi}_{\mathrm{visc} U}$ but now in the $\phi$ basis. On the other hand, for the inviscid modes the conditions are

$$
\Pi_{\text {inv }} \phi_{, x}=\Pi_{\text {inv }} \phi=\mathbf{0} \text { at } x=-L,
$$

where, analogously to (53.b) and (55.a) $\boldsymbol{\Pi}_{\text {inv }}$ is defined as

$$
\boldsymbol{\Pi}_{\text {inv }}=\left[\begin{array}{ll}
\mathbf{I}_{N_{\text {inv }} \times N_{\text {inv }}} & \mathbf{0}_{N_{\text {inv }} \times N_{\text {visc }}}
\end{array}\right] \mathbf{S}^{-1}
$$

and none at $x=L$ (Fig. 9). In total, we have $N_{\text {slab }}+N_{\text {inv }}$ equations upstream and $N_{\text {slab }}-N_{\text {inv }}$ downstream, making a total of $2 N_{\text {slab }}$ which is correct, since we have a system of $N_{\text {slab }}$ second-order ODEs.

The $\mathbf{F}_{\text {abs }}$ matrix is full, so that the ODEs are fully coupled at the boundaries. This is a characteristic of higher order absorbing boundary conditions, as the DtN [13]. "Nonlocal" in the DNL acronym, stands for this fact. 


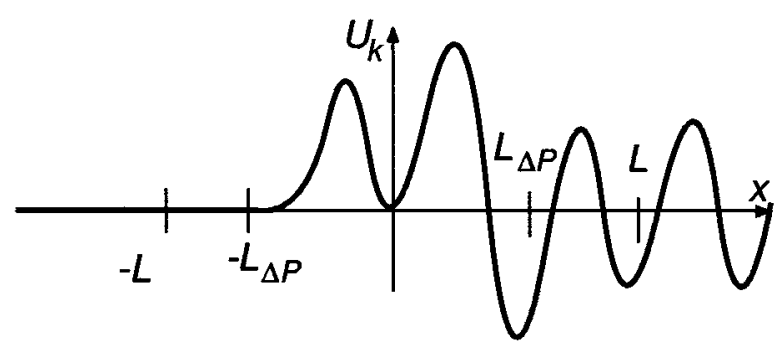

FIG. 9. Mode amplitude for the inviscid modes.

\section{DISCRETE RADIATION BOUNDARY CONDITION}

Now we will consider the full discretization of the wave-resistance problem for a pressure perturbation on a 1D structured grid. We mean by this a mesh which is composed of identical layers of nodes on planes at positions $x_{J}=J \Delta x$. The generating mesh in the channel section can be nonstructured and completely general. Afterwards, we will see that the extension to the general case, with a ship, or any other kind of obstacle, and a nonstructured mesh around it is very simple, provided that the mesh is 1D-structured far downstream and far upstream $(|x|>L)$ for a certain $L$.

The obvious way is to proceed to a $1 \mathrm{D}$ discretization of (12) by finite elements and a second-order approximation to Eqs. (54), (56), (57). This is straightforward, is briefly described in the next section, and will be called in the following the "partially discrete" approach. Another possibility is to discretize the interior governing equation (12) and then to design an absorbing boundary condition for the resulting system of difference equations. This is explained in Section 6.2, where it is explained in the context of the Helmholtz equation and the extension to potential flow with free surface is summarized. We term this approach the "fully discrete" one. It turns out to be that 1D discretization and the design of the absorbing boundary condition "do not commute" and the resulting discrete absorbing boundary conditions are different (see Fig. 11). We will show that the partially discrete alternative gives some amount of reflection due to the numerical error introduced in the wave number, and then, the fully discrete alternative is strongly recommended.

\subsection{Partially Discrete Approach}

Finite element 1D discretization of (12) gives

$$
\mathbf{A} \phi^{J+1}-2 \mathbf{B} \phi^{J}+\mathbf{A} \phi^{J-1}=\Delta x^{2} \tilde{\mathbf{G}}^{J},
$$

where

$$
\begin{aligned}
\mathbf{A} & =\tilde{\mathbf{M}}-\frac{1}{6} \Delta x^{2} \mathbf{K} \\
\mathbf{B} & =\tilde{\mathbf{M}}+\frac{1}{3} \Delta x^{2} \mathbf{K} \\
\tilde{\mathbf{G}}^{J} & =\frac{1}{6}\left(\mathbf{G}^{J+1}+4 \mathbf{G}^{J}+\mathbf{G}^{J-1}\right) .
\end{aligned}
$$

The boundary conditions (54), (56), can be discretized with a second-order approximation to the first derivative by means of a fictitious layer (see Fig. 10). Let $N$ be the last node 


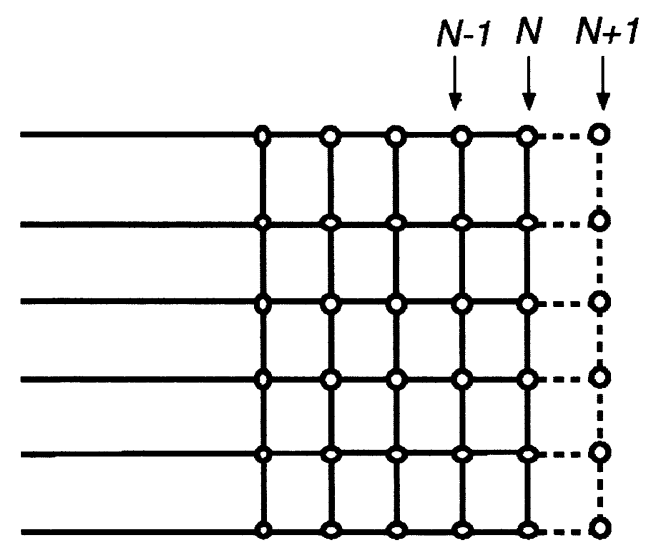

FIG. 10. Second-order approximation to the artificial boundary condition.

layer of the mesh (so that $x_{N}=L$ ) and let $N+1$ be the fictitious layer; then the discrete version of the boundary condition is

$$
\begin{aligned}
\boldsymbol{\Pi}_{\mathrm{visc}}\left(\frac{\phi^{N+1}-\phi^{N-1}}{2 \Delta x}+\mathbf{F}_{\mathrm{abs}} \phi^{N}\right) & =\mathbf{0}, \\
\mathbf{A} \phi^{N+1}-2 \mathbf{B} \phi^{N}+\mathbf{A} \phi^{N-1} & =\mathbf{0}
\end{aligned}
$$

where we added the "interior" governing equation for $J=N$ in order to balance the number of equations and unknowns. Note that we assumed $\tilde{\mathbf{G}}^{N}=\mathbf{0}$ due to (8). Similarly, at the left end we have

$$
\begin{aligned}
\boldsymbol{\Pi}_{\mathrm{visc}}\left(\frac{\phi^{-N+1}-\phi^{-N-1}}{2 \Delta x}-\mathbf{F}_{\mathrm{abs}} \phi^{-N}\right) & =\mathbf{0}, \\
\mathbf{A} \phi^{-N+1}-2 \mathbf{B} \phi^{-N}+\mathbf{A} \phi^{-N-1} & =\mathbf{0}, \\
\boldsymbol{\Pi}_{\mathrm{inv}} \phi^{-N} & =\mathbf{0}, \\
\boldsymbol{\Pi}_{\mathrm{inv}}\left(\frac{\phi^{-N+1}-\phi^{-N-1}}{2 \Delta x}\right) & =\mathbf{0} .
\end{aligned}
$$

Do not confuse the order of approximation to the semi-discrete boundary conditions (54), (56) with the order of the boundary condition itself. Usually, absorbing boundary conditions are classified as first order, second order, etc., depending on the order of expansion with respect to the angle of incidence of a plane wave with respect to the normal to the boundary. In this respect, this boundary condition is exact like the DtN in the sense that it is fully absorbent under mesh refinement.

\subsection{Fully Discrete Approach}

Now consider again the discrete Helmholtz equation as described in Section 4.2, whose absorbing boundary condition is (38). Discretization by FEM on a mesh of constant mesh step $\Delta x$ gives

$$
\frac{\phi^{j+1}-2 \phi^{j}+\phi^{j-1}}{\Delta x^{2}}-\frac{1}{6} \lambda\left(\phi^{j+1}+4 \phi^{j}+\phi^{j-1}\right)=0 .
$$


Second-order discretization of the absorbing boundary condition about $x_{N}$ with a fictitious node $N+1$ leads to

$$
\begin{gathered}
\frac{\phi^{N+1}-\phi^{N-1}}{2 \Delta x}-i k \phi^{N}=0, \\
\frac{\phi^{N+1}-2 \phi^{N}+\phi^{N-1}}{\Delta x^{2}}-1 / 6 \lambda\left(\phi^{N+1}+4 \phi^{N}+\phi^{N-1}\right)=0,
\end{gathered}
$$

where $N+1$ corresponds to the fictitious node. The interior equation (64.b) for the node $N$ equation is added in order to balance the number of equations and unknowns. Eliminating the potential at the fictitious node,

$$
\phi^{N}=f_{\mathrm{PD}} \phi^{N-1}
$$

where

$$
f_{\mathrm{PD}}=\left(\frac{1-\frac{1}{3} \lambda \Delta x^{2}}{1+\frac{1}{6} \lambda \Delta x^{2}}-i k \Delta x\right)^{-1} .
$$

The "fully discrete" alternative shown in Fig. 11 is to find the general solution to the difference equation (63) in the form

$$
\phi_{j}=a \mu_{+}^{j}+b \mu_{-}^{j},
$$

where $\mu_{ \pm}$are solutions of the characteristic equation

$$
A \mu^{2}-2 B \mu+A=0
$$

with

$$
A=\left(1+\frac{1}{6} k^{2} \Delta x^{2}\right), \quad B=\left(1-\frac{1}{3} k^{2} \Delta x^{2}\right) .
$$

Both $\mu_{ \pm}$are complex conjugate and of unit modulus; see Fig. 13. The perturbation analysis shows that $\mu_{+}$moves inside the unit circle for a small dissipative term, whereas $\mu_{-}$moves outside, so that we retain the $\mu^{+}$component in (67) and satisfy the boundary condition

$$
\phi^{N}=f_{\mathrm{FD}} \phi^{N-1}
$$

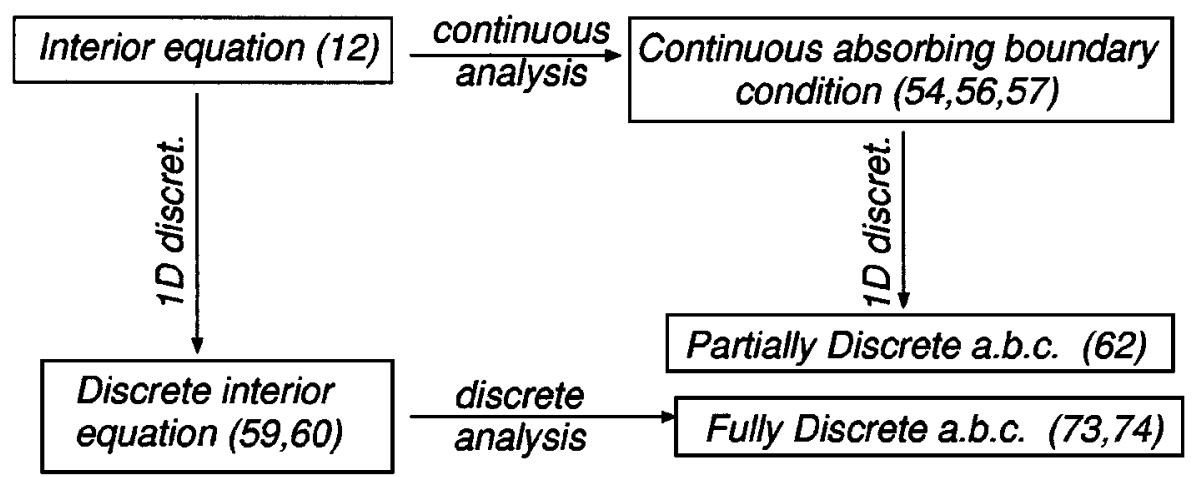

FIG. 11. Schematic description of the "partially" and "fully" discrete versions of the boundary condition. 


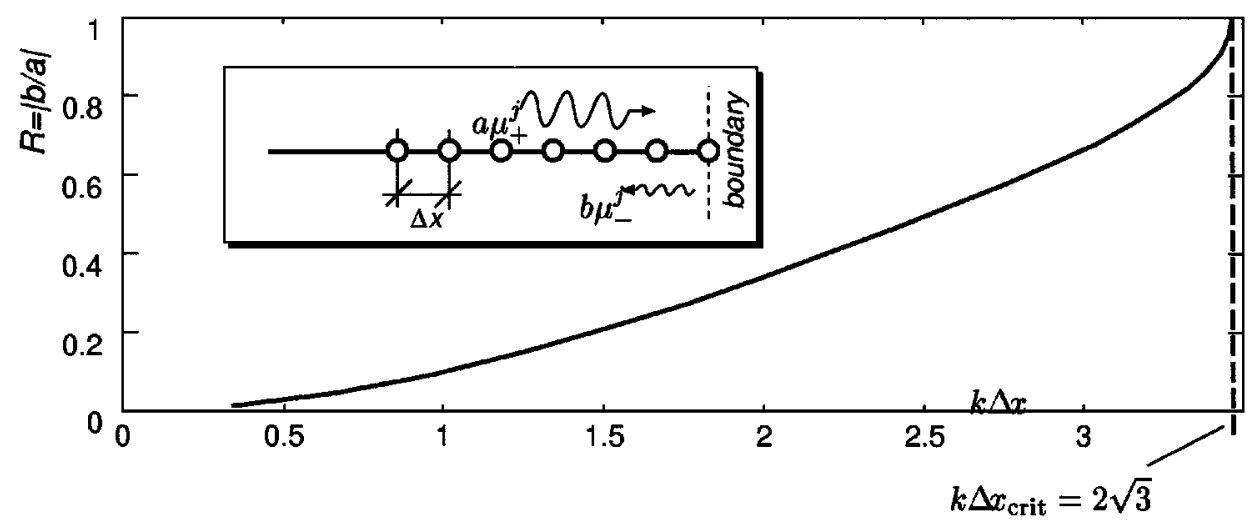

FIG. 12. Discrete coefficient of reflection for the 1D Helmholtz equation.

where

$$
f_{\mathrm{FD}}=\mu^{+} .
$$

Now, in order to compute the reflection coefficient for a given boundary condition we insert the general solution (67) in the boundary condition in the form (65) or (70) and obtain a linear relationship between the amplitude of the incoming wave $b$ to the outgoing wave $a$ :

$$
R=\left|\frac{b}{a}\right|=\left|\frac{\mu_{+}^{N}}{\mu_{-}^{N}} \frac{1-f \mu_{+}^{-1}}{1-f \mu_{-}^{-1}}\right|=\left|\frac{1-f \mu_{+}^{-1}}{1-f \mu_{-}^{-1}}\right| .
$$

It is clear now from this and (71) that the fully discrete boundary condition gives a null reflection coefficient, whereas the partially discrete one (65), (66) suffers from some amount of reflection which is computed from (72), (66) and plotted in Fig. 12. This reflection is purely numerical $R \propto(k \Delta x)^{2}$ for small $k \Delta x$, and for the critical wave number, where the wave becomes evanescent, it reaches the maximum value of $100 \%$ reflection. The "fully

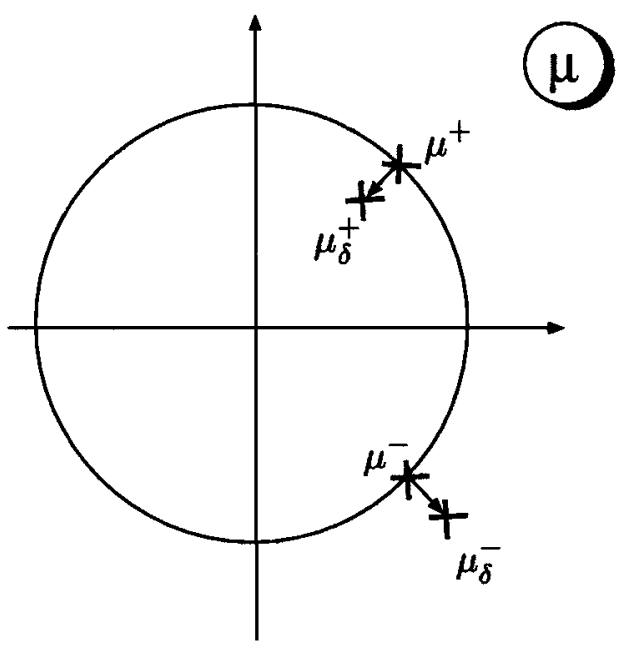

FIG. 13. Roots of the characteristic discrete equation. 
discrete" approach is then adopted. The term "discrete" in the name of the method stands for this particular way of obtaining the absorbing boundary condition by the straightforward solution of the system of discrete equations.

In conclusion, the "fully discrete" version of the absorbing boundary condition for the potential flow problem with a free-surface is given by

$$
\begin{aligned}
\boldsymbol{\Pi}_{\mathrm{visc}}\left(\phi^{N}-\mathbf{F}_{\mathrm{FD}} \phi^{N-1}\right) & =\mathbf{0}, \\
\boldsymbol{\Pi}_{\mathrm{visc}}\left(\phi^{-N}-\mathbf{F}_{\mathrm{FD}} \phi^{-N+1}\right) & =\mathbf{0}, \\
\boldsymbol{\Pi}_{\mathrm{inv}} \phi^{-N}=\boldsymbol{\Pi}_{\mathrm{inv}} \phi^{-N+1} & =\mathbf{0},
\end{aligned}
$$

where

$$
\mathbf{F}_{\mathrm{FD}}=\mathbf{S} \operatorname{diag}\{\underbrace{0, \ldots, 0}_{N_{\text {inv }}}, \underbrace{\mu_{N_{\text {inv }}+1}^{+}, \ldots, \mu_{N_{\text {slab }}}^{+}}_{N_{\text {visc }}}\} \mathbf{S}^{-1},
$$

and

$$
\left.\begin{array}{rl}
\mu_{k}^{+} & =\xi_{k}-\sqrt{\xi_{k}^{2}-1} \\
\xi_{k} & =\frac{1+\frac{1}{3} \Delta x^{2} \lambda_{k}}{1-\frac{1}{6} \Delta x^{2} \lambda_{k}}
\end{array}\right\} \text { for } k=N_{\mathrm{inv}}+1, \ldots, N_{\text {slab }}
$$

\section{IMPLEMENTATION DETAILS}

\subsection{Nonstructured Meshes and Nonconstant Base-Flow Velocity Vector}

The method was described for structured grid and constant base-flow in the $x$ direction, but it only needs these restrictions to apply several layers near the upstream and downstream boundaries (see Fig. 14), allowing a nonstructured mesh and nonconstant flow in a certain region near the ship. Once those layers are available, the matrices $\mathbf{A}$ and $\mathbf{B}$ can be evaluated and $\mathbf{F}_{\mathrm{FD}}$ and $\boldsymbol{\Pi}_{\mathrm{visc}}$ are computed in order to impose conditions (73), (74).

\subsection{Numerical Computation of the Eigen-Decomposition (16)}

In practice, we found it preferable to compute the absorption matrix directly by eigendecomposition of $\mathbf{A}^{-1} \mathbf{B}$ (rather than with $\mathbf{M}^{-1} \mathbf{K}$ ). A standard FEM code (with standard boundary conditions, say Neumann, at the inlet/outlet planes) computes the matrix system with the free surface term. As no numerical dissipation term is added, the free surface term can be easily cast in a weak form. (This point is important for the finite element formulation). Matrices $\mathbf{A}$ and $\mathbf{B}$ are extracted, and $\mathbf{A}^{-1} \mathbf{B}$ and its eigen-decomposition is computed as a full matrix. This works rather as a black box on matrices A and $\mathbf{B}$. The term "algebraic" for the method stands for this. We used the standard LAPACK routines DGECO, DGESL, and DGEEVX to perform inversions and eigen-decompositions. As the size of the matrices is one dimension lower, this part of the computations is not relevant regarding CPU time and RAM requirements.

\subsection{FEM System Solution; Effects of Shifting the Boundary Conditions}

Regarding the additional cost when solving the global FEM system matrix by a typical direct skyline solver, note that the resulting absorbing matrix is full, i.e. connecting all the 


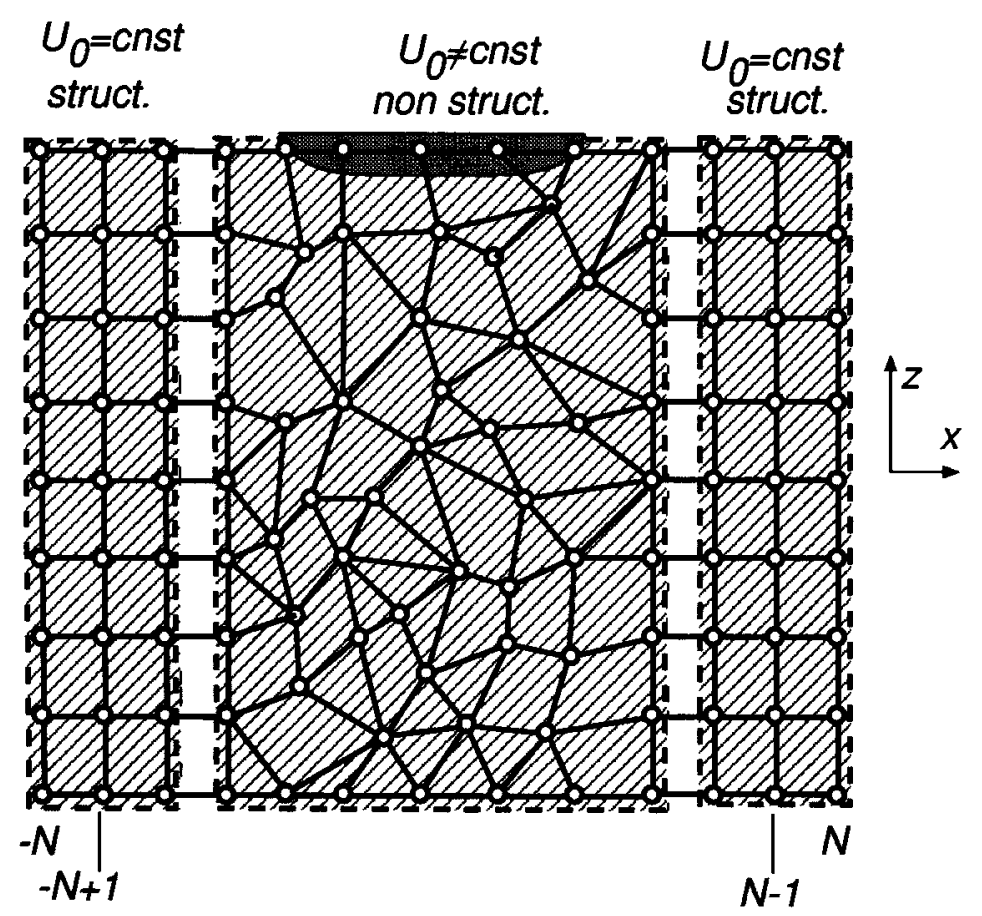

FIG. 14. Unstructured mesh with structured layers upstream and downstream.

degrees of freedom on the two first structured layers (marked as $-N$ and $-N+1$ in Fig. 14), and also on the last two (marked as $N-1$ and $N$ ). This represents some local increase in the bandwidth but the overall increase in RAM requirement is negligible.

However, there is a practical problem related to the shifting of the boundary conditions from the downstream boundary to the upstream one (see Fig. 15). Considering a mesh like that in Fig. 14, the degrees of freedom are numbered from left to right. At the left in Fig. 15 we see the symmetric structure of the matrix for the problem with standard Neumann boundary conditions at the left and right boundaries. Then the proposed boundary conditions imply the deletion of some rows corresponding to the inviscid modes downstream and adding
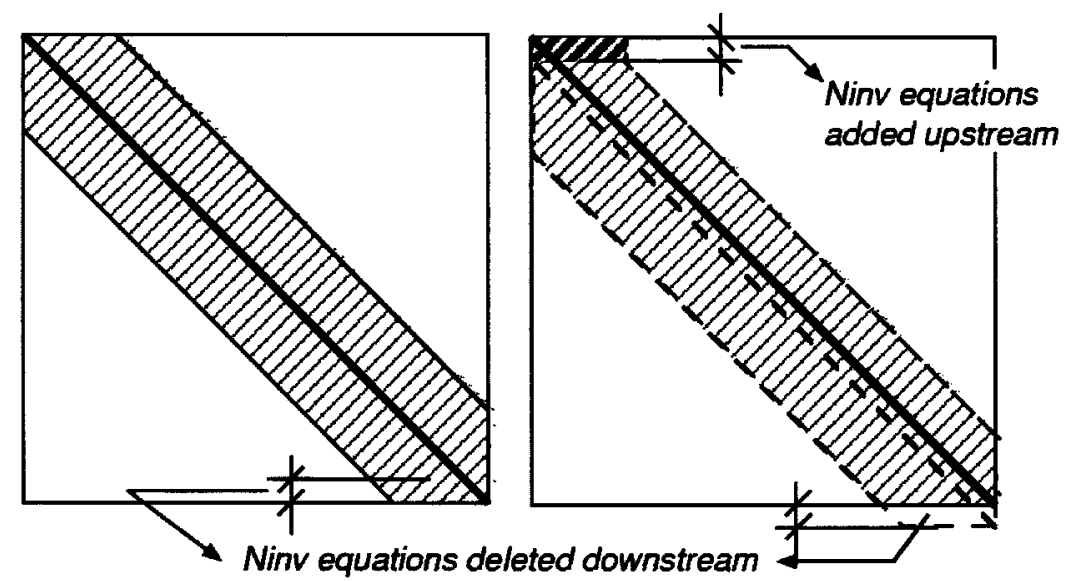

FIG. 15. Effect of shifting boundary conditions from downstream to upstream. 
the same number of rows upstream. There are two possibilities, replacing the deleted rows by the new equations and leaving the ordering of the equations unaltered, or putting the new equations on top. This last possibility is shown in the figure. Note that this causes a shift of the main diagonal of the system matrix and it is likely that some elements in the new diagonal could be null, which in turns suggests that some null pivots could be found during elimination. The first possibility has the same drawback. Another alternative is to solve the original system which, in addition, is symmetric, taking into account the fact that $N_{\text {inv }}$ equations have been modified by adding an unknown right-hand side. The system is solved for $N_{\text {inv }}$ right-hand sides with each one having unity in the corresponding modified equation and zero in the others. The solution is then found as a linear combination of these solutions and imposing the relaxed equations on this combination. This leads to a $N_{\text {inv }} \times N_{\text {inv }}$ system of linear equations that determines the coefficients in the expansion. We call this method "superposition" and has the advantage that the cost and structure of the solution for each of the $N_{\text {inv }}$ right-hand sides is the same as a standard finite elements problem with standard boundary conditions, like the Laplace operator, for instance. Moreover, the proposed boundary conditions can be put in such way that the resulting matrix is symmetric, with a significant reduction in the RAM requirement. However, the system to be solved is singular for a certain discrete set of Froude numbers. In practice, one computes the drag or wave pattern for a certain set of Froude numbers, and it is unlikely that one of these would coincide with one of the singular Froude numbers. Details of this are described in [5]. See also the numerical example for the submerged cylinder [Section 8.1.3].

\subsection{Wave Resistance Calculation}

The energy spent by the ship against the wave-resistance is emitted in the form of gravity waves that form the wave pattern. This means that we can compute the drag by integrating the momentum flux density on a plane section downstream and this is the form in which the wave resistance is separated from the total resistance in experiments (see [7]). After some manipulation, it can be shown [4-6] that the expression is

$$
F_{x}=-\frac{1}{2} \rho \int_{\Sigma_{\text {out }}}\left(\phi_{, x}^{2}-\phi_{, y}^{2}-\phi_{, z}^{2}\right) d S+\frac{1}{2} \rho g \int_{y=-L_{y}}^{+L_{y}} \eta\left(x_{\text {out }}, y, 0\right)^{2} d y,
$$

and the discrete version is

$$
F_{x}=\frac{1}{2} \rho \sum_{j=1}^{N_{\text {inv }}} b_{j}^{2}\left(\phi_{j}^{\mathrm{T}} \mathbf{K} \phi_{j}\right)
$$

where $\phi_{j}$ is the $j$ th eigenvector of system (16), i.e. the $j$ th column of matrix $\mathbf{S}$, and $b_{j}$ is the "amplitude" for the $j$ th inviscid mode, defined by

$$
U_{j}=b_{j} \sin \left(\sqrt{\left|\lambda_{j}\right|} x+\gamma_{j}\right)
$$

where $\gamma_{j}$ are arbitrary phases. It is obvious from this expression that it is independent of the position of the boundary and that a positive drag is always assured. This is a great advantage over Dawson-like methods based on numerical viscosities, which compute the wave-resistance by straightforward integration of pressure forces over the hull [3]. 


\section{NUMERICAL EXAMPLES}

\subsection{Two-Dimensional Examples}

8.1.1. Submerged dipole (infinitesimal cylinder). The problem of a submerged cylinder has an analytical solution in the limit of very small radius, in which case the cylinder can be replaced by a dipole (see Fig. 16). Moreover, the drag calculation has been performed by computing the perturbation in pressure produced by the submerged dipole without free surface and applying this extra pressure as an equivalent hovercraft problem. The extra pressure is

$$
\Delta P(x)=-2 \rho U_{\infty}^{2} b^{2} \operatorname{Re}\left\{\frac{1}{(x+i f)^{2}}\right\},
$$

where $f$ is the depth of the cylinder and $b$ is its radius (here it enters only through the intensity of the dipole). The analytical expression for the drag is $[6,22]$

$$
C_{\mathrm{w}}=4 \pi^{2}(b / f)^{3} \mathrm{Fr}^{-6} e^{-2 / \mathrm{Fr}^{2}},
$$

where $\mathrm{Fr}$ is the Froude number based on depth; i.e., $\mathrm{Fr}=U_{\infty} / \sqrt{g f}$. In the figure we plot $(b / f)^{3} C_{\mathrm{w}}$ which depends only on Fr. This is an interesting case in the sense that the pressure perturbation does not have compact support; i.e. it extends to infinity. However, it decays $\propto|x|^{-2}$, and we see that the agreement is good. The mesh was structured with

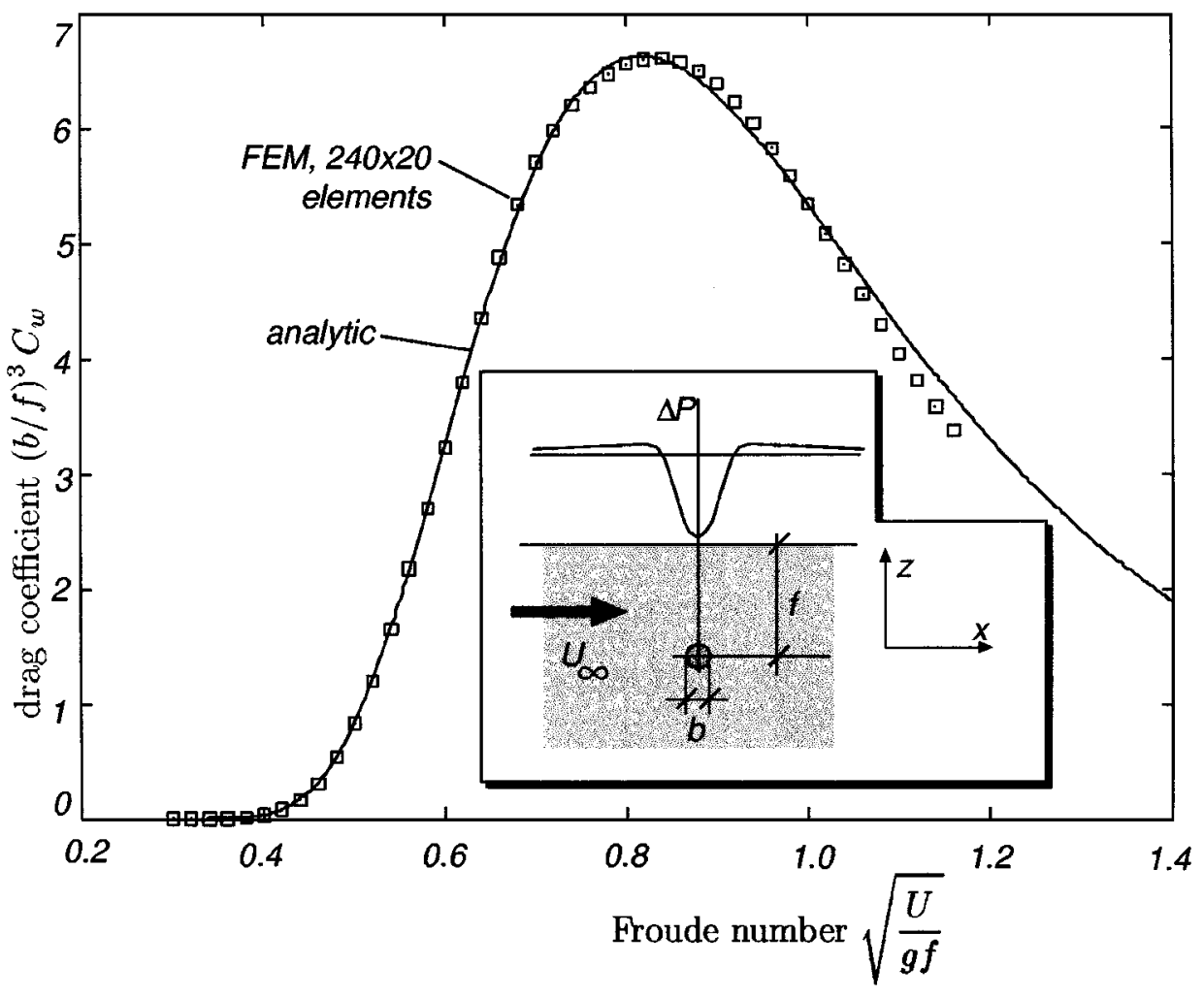

FIG. 16. Drag curve for the submerged dipole (cylinder with diameter $b \ll f$ ). The drag coefficient is normalized to $b / f=1$. 


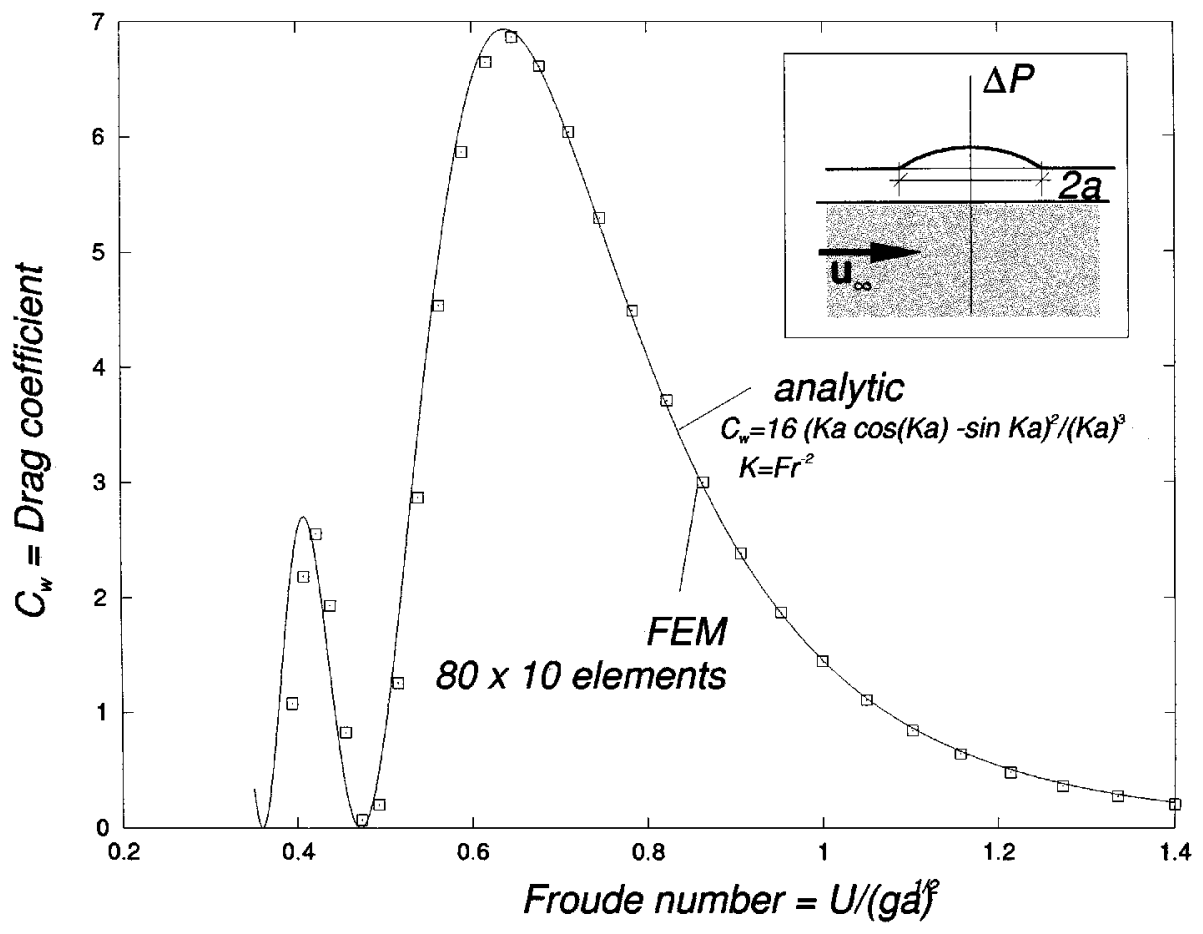

FIG. 17. Drag curve for the parabolic pressure distribution.

$2 \times 240(x) \times 20(z)$ triangular elements covering the rectangle $|x|<6,-3<z<0(f=1)$. The mesh was refined near the surface in such a way that $\Delta z_{\text {bottom }} / \Delta z_{\text {surface }}=10$.

8.1.2. Parabolic pressure distribution. This is another $2 D$ example, for a prescribed pressure distribution of the form

$$
\Delta P= \begin{cases}1-(x / a)^{2} & \text { for }|x|<a \\ 0 & \text { for }|x|>a\end{cases}
$$

The analytical drag coefficient is [6]

$$
C_{\mathrm{w}}=16 \frac{(K a \cos K a-\sin K a)^{2}}{(K a)^{3}},
$$

where $K a=1 / \mathrm{Fr}^{2}$, and the Fr number is taken based on $a$ (see Fig. 17).

8.1.2.1. Invariance of the discrete solution with boundary position. This pressure distribution has compact support and, then, we can check that the solution is independent of the position of the boundary condition, as was asserted at the end of Section 6. To verify this, we modeled the problem at $\mathrm{Fr}=0.8$ with two FEM meshes. The first one has $2 \times 80 \times 10$ triangular elements with $\Delta x=$ const and $\Delta z_{\text {bottom }} / \Delta z_{\text {surface }}=10$, covering the region $-6<x<2,-3<z<0$. The second one is identical to this one, but it has been prolonged downstream to $x=6$ with 40 additional element layers, keeping the same $\Delta x$. We can see in Fig. 18 the potential on the free surface versus $x$ for both meshes. Both coincide to machine precision in the overlapping region. 


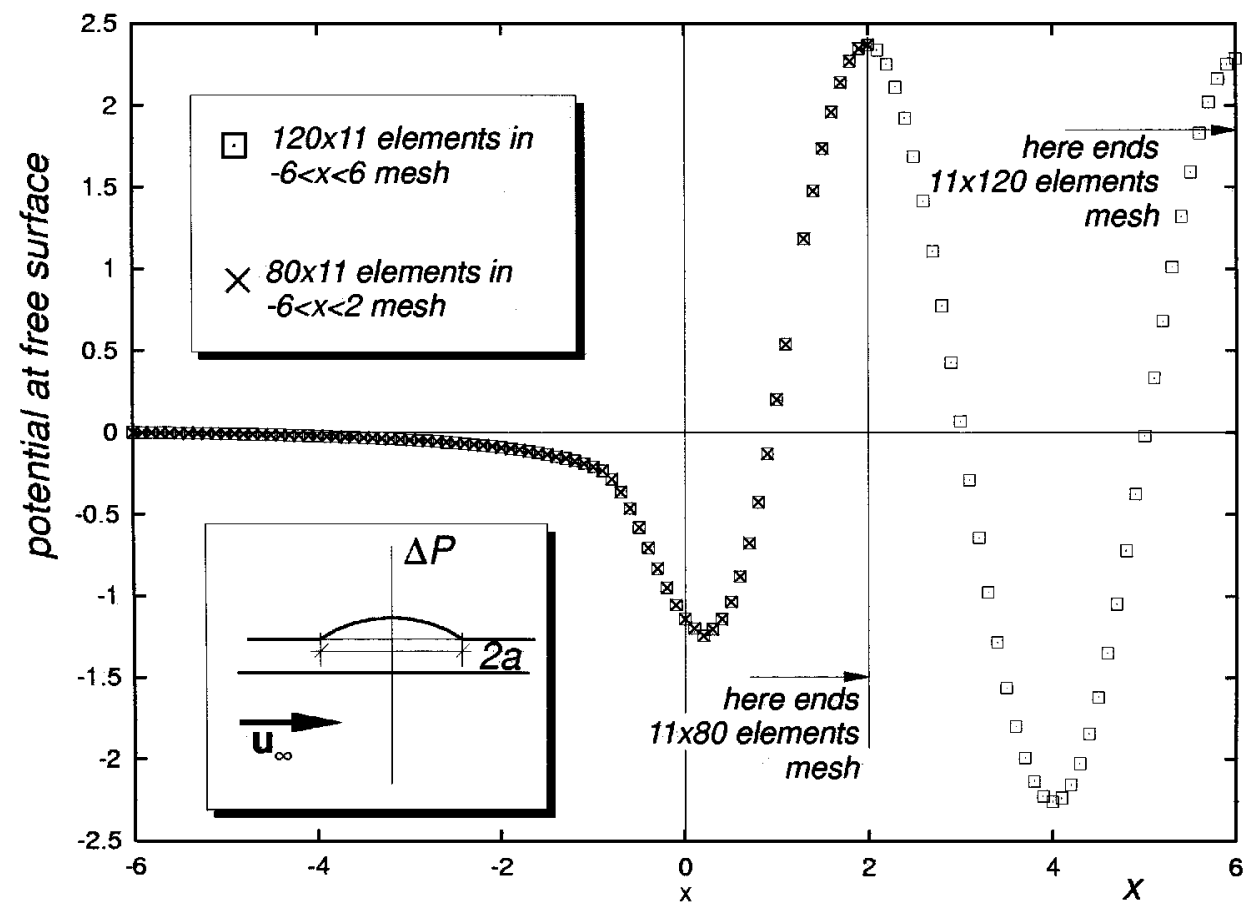

FIG. 18. Invariance of the discrete solution with the position of the absorbing boundary condition.

8.1.2.2. Constancy of elevation. In $2 \mathrm{D}$ problems, the trailing elevation wave is a sinusoid of constant amplitude far downstream. Numerical viscosity damps this wave, and then constancy of the trailing wave amplitude is a measure of the precision of the method. In Fig. 19, we can see the trailing wave for the parabolic pressure distribution for $a=1$ at $\mathrm{Fr}=0.5$ for a mesh similar to the previous ones, but extended to $x=10$. No damping is observed qualitatively. A detailed analysis shows that the nodal amplitudes remain constant to machine precision.

8.1.3. Submerged cylinder. This is the case of a cylinder of finite radius $b$ at a depth $f=4 b$ to its axis in a channel of depth $H=2 f$ (see Fig. 20). The mesh is a typical "O" mesh around the cylinder, with two additional structured layers at the inlet and outlet planes. The purpose of this numerical example is to show a case where the mesh is not fully structured. The drag coefficient has been normalized with a factor $(b / f)^{3}$, to make it comparable with the infinitesimal radius (dipole) case. The resulting drag curve is very similar to that one for a dipole. The drag was computed for 500 values of $\mathrm{Fr}$ in the range $0.5<\mathrm{Fr}<1$ and we show also the condition number of the linear system (see Fig. 21). We see that, as discussed in Section 7.3, the system is singular at a discrete set of Froude numbers. For very small Froude numbers the condition number growths indefinitely. We do not have a satisfactory explanation for this, but we stress the fact that the Froude number range for which we obtain valid results covers the range of practical interest and is wider than those for other methods.

\subsection{D Examples}

8.2.1. Wigley hull. The drag curve for the Wigley model $1805 \mathrm{~A}$ is shown in Fig. 22 . The hull shape for this model is defined by $y= \pm\left(1-x^{2} / 64\right)\left(1-0.6 x^{2} / 64\right)\left(1-z^{2}\right)$ for 


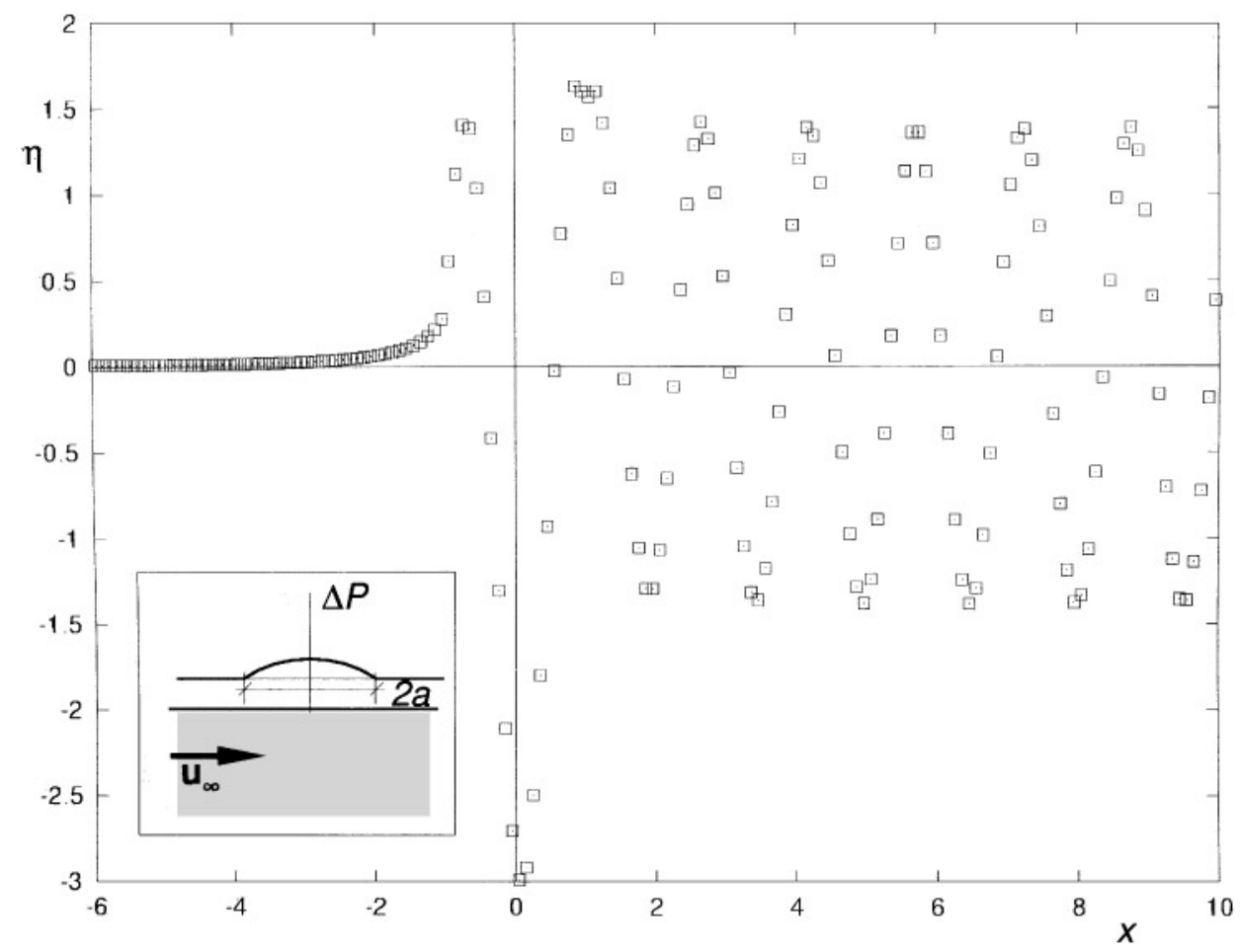

FIG. 19. Free surface elevation showing that the amplitude of the trailing wave is not damped.

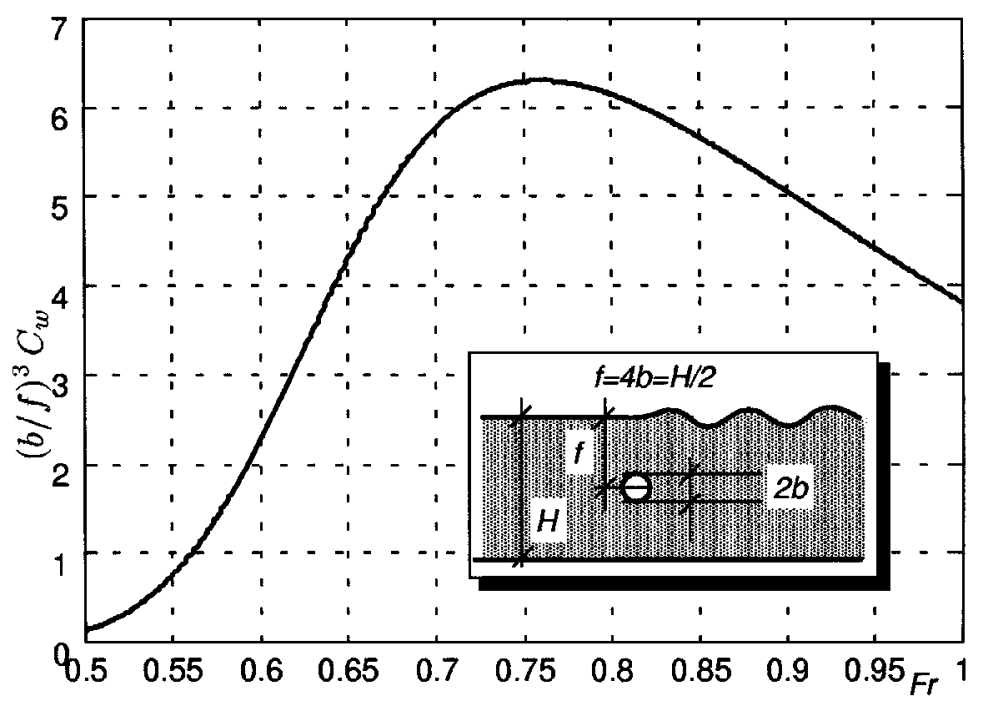

FIG. 20. Wave resistance coefficient for a submerged cylinder in a channel of finite depth. 


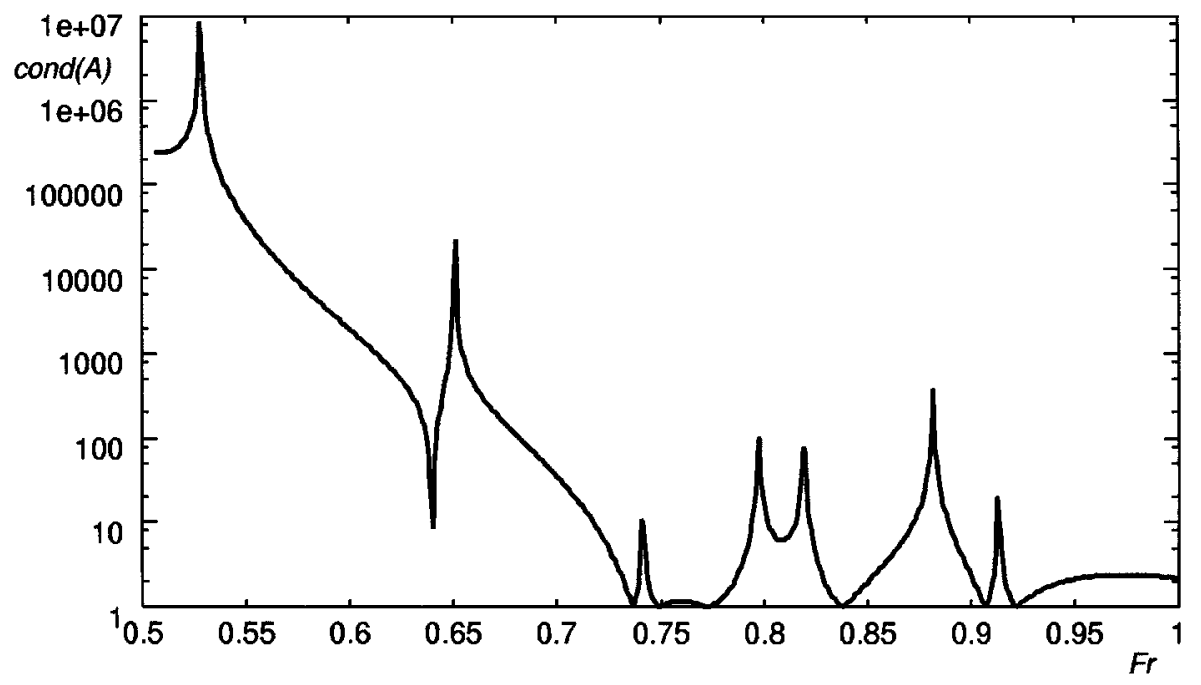

FIG. 21. Condition number for the submerged cylinder.

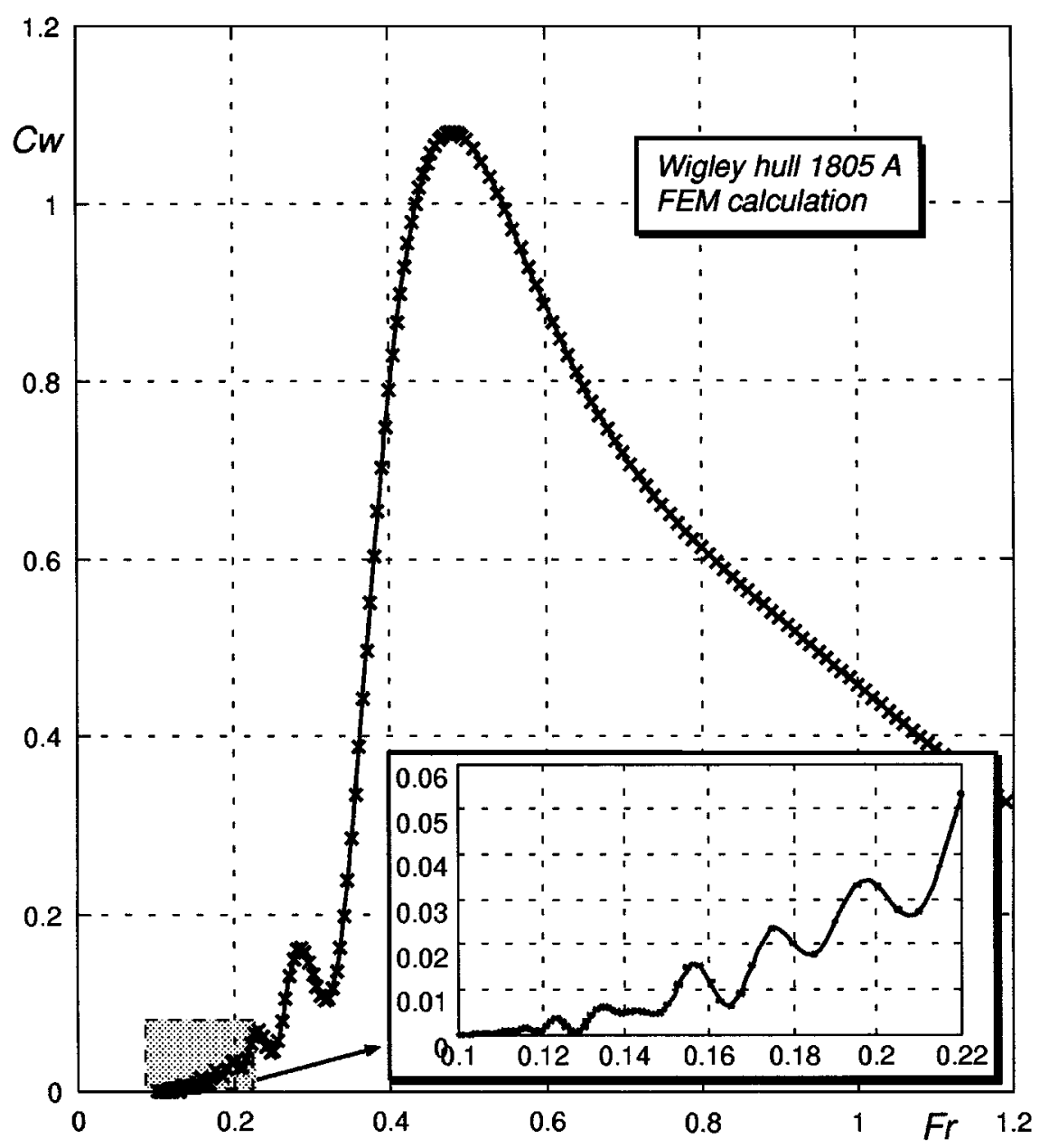

FIG. 22. Drag curve for the Wigley hull. 


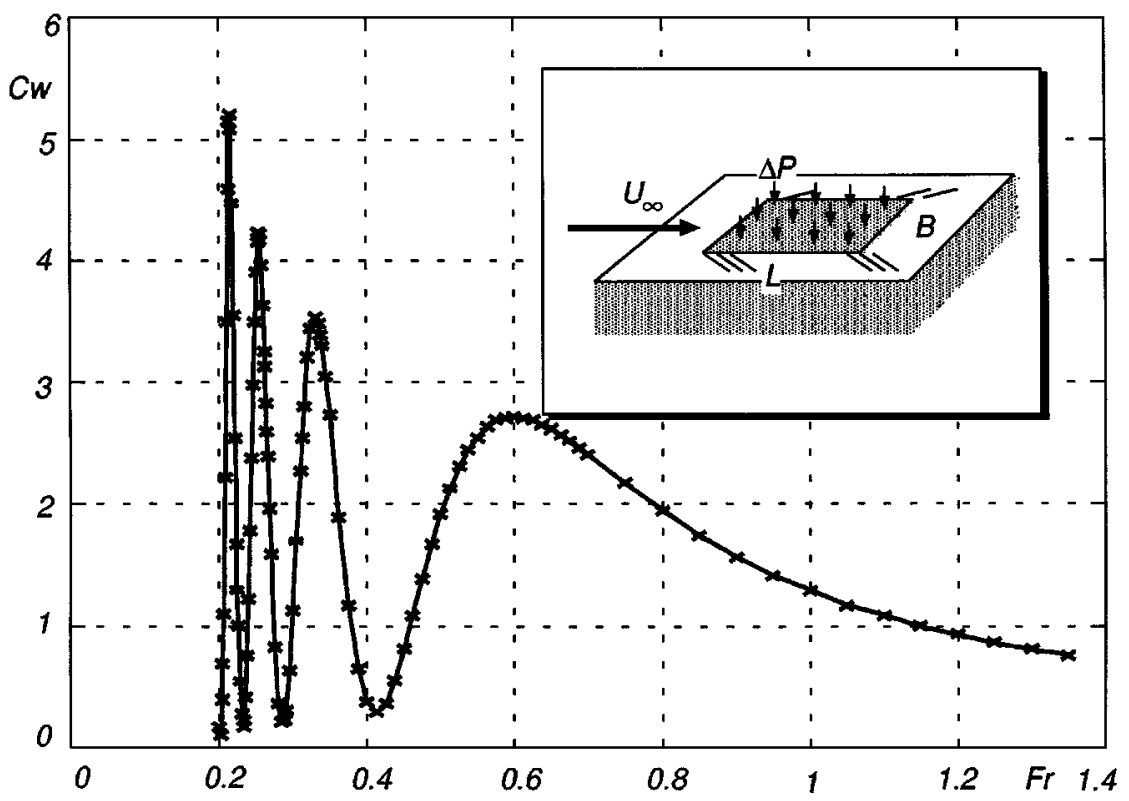

FIG. 23. Drag curve for the rectangular pressure distribution.

$|x|<8, z>-1$. The drag coefficient is defined as

$$
C_{\mathrm{w}}=\frac{250}{\pi} \frac{F_{x}}{\Omega_{\text {ship }}^{2 / 3} \rho U_{\infty}^{2}},
$$

where $\Omega_{\text {ship }}=4 \frac{52}{75}$ is the volume of the ship. The FEM mesh had $50(x) \times 13(y) \times 13(z)=$ 8450 elements, and the result is in good agreement with results found in the literature $[1,7]$. Note that a whole set of secondary maxima is cleanly captured, extending to Froude as low as 0.1 . In the other extreme, Froude numbers as high as 1.2 are computed without problems, whereas standard methods like those derived from Dawson suffer from reflections, especially at high Froude numbers.

8.2.2. Rectangular pressure distribution. We consider a uniform rectangular pressure distribution of width $B$ and length $L$, such that $L / B=\frac{3}{2}$, for which experimental and analytical results are reported in [7]. This case is interesting, since it is purely $3 \mathrm{D}$ and large oscillations in the drag curve at small Froude numbers are expected, due to the discontinuity in the pressure distribution. The mesh had $30(x) \times 15(y) \times 10(z)=4500$ elements (see Fig. 23). Coincidence with results reported in [7] are very good. Whereas only the maximum around $\mathrm{Fr}=0.33$ is shown in those results, we here capture two additional maxima at $\mathrm{Fr}=0.215$ and 0.255 , approximately.

\section{CONCLUSIONS}

A discrete nonlocal (DNL) absorbing boundary condition for the wave resistance problem has been presented. It is based on an eigen-decomposition of the system of ODEs that results from partial discretization in the transversal section of the governing equations. By construction, the numerical solution is independent of the position of the absorbing 
boundary. Even if this is a well-known result for exact boundary conditions on a wide range of problems such as Helmholtz equations, elasticity equations in the frequency domain or the sea-keeping problem [17], it has not been reported in the literature for the specific case of the wave-resistance problem. As no numerical viscosity is used, the wave-resistance can be computed from a momentum flux balance and positive wave resistances are guaranteed. Drag curves computed with this method exhibit very well-defined secondary maxima, and computations can be carried out for a wide range of Froude numbers.

\section{APPENDIX 1: NEUMANN BOUNDARY CONDITION AT THE BOTTOM}

Considering now the case of Neumann boundary condition at the bottom, then it is easy to show that the associated matrix for the Laplace operator on a typical section is only semidefinite positive, since the vector

$$
\mathbf{v}_{1}=c\left[\begin{array}{llll}
1 & 1 & \ldots & 1
\end{array}\right]
$$

representing a constant potential field

$$
v_{1}(y, z)=\sum_{j} v_{1 j} N_{j}(y, z)=c,
$$

is an eigenvector with null eigenvalue for $\mathbf{K}$, i.e.,

$$
\mathbf{K} \mathbf{v}_{1}=0
$$

In order to have a unit vector we choose $c=N_{\text {slab }}^{-1 / 2}$. This is the only eigenvector with null eigenvalue of $\mathbf{K}$, so that completing to an orthonormal basis $\left\{\mathbf{v}_{j}\right\}_{j=1}^{N_{\text {slab }}}$

$$
\mathbf{V}^{T} \mathbf{V}=\mathbf{I}, \quad \mathbf{V}=\left[\begin{array}{llll}
\mathbf{v}_{1} & \mathbf{v}_{2} & \ldots & \mathbf{v}_{N_{\text {slab }}}
\end{array}\right],
$$

and making the change of variables

$$
\phi=\mathbf{V} \psi
$$

system (12) reads now

$$
\tilde{\mathbf{M}}_{\psi} \psi_{, x x}-\mathbf{K}_{\psi} \psi=\mathbf{G}_{\psi}(x),
$$

where the $\psi$ subindex stands for matrices and vectors in that basis. Matrices and vectors are block split in the subspace " 0 " spanned by the null eigenvector $\mathbf{v}_{1}$ and the subspace " + " spanned by the rest $\left\{\mathbf{v}_{j}\right\}_{j=2}^{N_{\text {slab }}}$, so that

$$
\begin{aligned}
\mathbf{K}_{\psi} & =\mathbf{V}^{-1} \mathbf{K V}=\left[\begin{array}{cc}
0 & \mathbf{0}_{1 \times N_{\text {slab }}} \\
\mathbf{0}_{N_{\text {slab }} \times 1} & \mathbf{K}_{\psi}^{++}
\end{array}\right], \\
\tilde{\mathbf{M}}_{\psi} & =\mathbf{V}^{-1} \tilde{\mathbf{M}} \mathbf{V}=\left[\begin{array}{cc}
\tilde{M}_{\psi}^{00} & \tilde{\mathbf{M}}_{\psi}^{0+} \\
\tilde{\mathbf{M}}_{\psi}^{+0} & \tilde{\mathbf{M}}_{\psi}^{++}
\end{array}\right]
\end{aligned}
$$


and

$$
\mathbf{G}_{\psi}=\mathbf{V}^{-1} \mathbf{G}=\left[\begin{array}{c}
G_{\psi}^{0} \\
\mathbf{G}_{\psi}^{+}
\end{array}\right]
$$

The $j, k$ th element of $\mathbf{K}_{\psi}$ is $\mathbf{v}_{j} \cdot \mathbf{K} \cdot \mathbf{v}_{k}$, so that its first column is null by (85). By symmetry, the first row is also null. Equation (88) in split form reads

$$
\begin{array}{r}
\tilde{M}_{\psi}^{00} \psi_{, x x}^{0}+\tilde{\mathbf{M}}_{\psi}^{0+} \psi_{, x x}^{+}=G_{\psi}^{0}, \\
\tilde{\mathbf{M}}_{\psi}^{+0} \psi_{, x x}^{0}+\tilde{\mathbf{M}}_{\psi}^{++} \psi_{, x x}^{+}-\mathbf{K}_{\psi}^{++} \psi^{+}=G_{\psi}^{+} .
\end{array}
$$

Assuming $\tilde{M}_{\psi}^{00} \neq 0$ (we will discuss this assumption later), we can eliminate $\psi^{0}$ and arrive at

$$
\tilde{\mathbf{M}}^{*} \psi_{, x x}^{+}-\mathbf{K}_{\psi}^{++} \psi^{+}=G^{*}
$$

which is an equation for $\psi^{+}$, where

$$
\begin{gathered}
\mathbf{M}^{*}=\tilde{\mathbf{M}}_{\psi}^{++}-\left(\tilde{M}_{\psi}^{00}\right)^{-1} \tilde{\mathbf{M}}_{\psi}^{+0} \tilde{\mathbf{M}}_{\psi}^{0+} \text { is symmetric } \\
\mathbf{K}_{\psi}^{++} \text {is symmetric and positive definite } \\
\mathbf{G}^{*}=\mathbf{G}_{\psi}^{+}-\left(\tilde{M}_{\psi}^{00}\right)^{-1} G_{\psi}^{0} \tilde{\mathbf{M}}_{\psi}^{+0} .
\end{gathered}
$$

Due to these properties, an eigenvalue decomposition like (16) applies for the pair $\mathbf{K}_{\psi}^{++}, \mathbf{M}^{*}$. The analysis for this reduced system follows as for the case of the null Dirichlet condition at the bottom and appropriate absorbing boundary conditions can be found. It remains to determine the appropriate boundary conditions for the $\psi^{0}$ mode. Coming back to (90.a) and solving for $\psi^{0}$, we obtain

$$
\tilde{M}_{\psi}^{00} \psi_{, x x}^{0}=G_{\psi}^{0}-\left(\tilde{\mathbf{M}}_{\psi}^{+0} \psi_{, x x}^{+}\right)
$$

whose solution is

$$
\psi^{0}=\left(\tilde{M}_{\psi}^{00}\right)^{-1}\left(\int_{0}^{x} \int_{0}^{x^{\prime}} G_{\psi}^{0}\left(x^{\prime \prime}\right) d x^{\prime \prime} d x^{\prime}-\tilde{\mathbf{M}}_{\psi}^{+0} \psi^{+}\right)+a x+b .
$$

The $b$ term represents a constant potential and can be dropped. The $a x$ term represents a potential constant on the channel section and varies linearly along the channel. This is equivalent to a change in the uniform base velocity compensated by a uniform raise in surface elevation, and it can also be dropped, so that no particular boundary condition has to be imposed for this "mode."

Now we discuss the implications of the restriction $\tilde{M}_{\psi}^{00} \neq 0$. It can be seen that $\tilde{M}_{\psi}^{00}=0$ when the channel is at a critical regime, i.e. when $\operatorname{Fr}_{H}=1$, because

$$
\begin{aligned}
\tilde{M}_{\psi}^{00} & =\sum_{j k=1}^{N_{\text {slab }}} \tilde{M}_{j k} v_{1 j} v_{1 k}, \\
& =\int_{\Sigma_{y z}} v_{1}^{2}(y, z) d y d z-K^{-1} \int_{\Sigma_{\text {free } 0}} v_{1}^{2}(y, 0) d y
\end{aligned}
$$


But $v_{1}(y, z)=$ const $=N_{\text {slab }}^{-1 / 2}$, so that

$$
\tilde{M}_{\psi}^{00}=\left(L_{y} / N_{\text {slab }}\right)\left(H-K^{-1}\right),
$$

and this is null when $\mathrm{Fr}_{H}=U_{\infty} /(g H)^{1 / 2}=1$. Usually, we are interested in the range $\mathrm{Fr}_{L}<1$, and $\operatorname{Fr}_{H}=(L / H)^{1 / 2} \mathrm{Fr}_{L}$, so that it is enough to take a mesh with $H$ several times larger than the ship length $L$, in order to have $\tilde{M}_{\psi}^{00} \neq 0$ in the range $\operatorname{Fr}_{L}<1$. Anyway, note that the analysis breaks down at a single Froude number, which proves the general applicability of the DNL methodology for deriving absorbing boundary conditions for a wide range of physical problems. Moreover, the fact that the decomposition proposed above breaks at $\mathrm{Fr}_{H}=1$ does not spoil the DNL methodology itself, since an alternative representation of the system may be found, appropriate for the DNL analysis. However, for reasons of space we will not discuss this point further here.

\section{APPENDIX 2: THE NUMBER OF "INVISCID” AND "VISCOUS” MODES}

For simplicity, we will consider the case of Dirichlet conditions at the bottom of the channel, so that matrix $\mathbf{K}$ is positive definite. Due to the Sylvester's inertia theorem [23] the number of positive and negative eigenvalues of system (16) remains the same if the matrix $\mathbf{K}$ is replaced by another positive definite symmetric matrix. We then replace $\mathbf{K}$ by $\mathbf{M}$ and let $\mathbf{W}$ and $\mathbf{B}$ be a solution for the eigenvalue problem

$$
\mathbf{M}_{\text {free }} \mathbf{W}=\mathbf{M W B}
$$

with $\mathbf{W}$ real and nonsingular and $\mathbf{B}$ real and diagonal, $\mathbf{B}=\operatorname{diag}\left\{\beta_{1}, \ldots, \beta_{N_{\text {slab }}}\right\} . \mathbf{M}_{\text {free }}$ is positive semi-definite and has rank $N_{\text {free }}$, where $N_{\text {free }}$ is the number of nodes in the slab that are on the free surface, so that there are $N_{\text {free }}$ positive eigenvalues and the rest is null; then we assume

$$
\begin{array}{ll}
\beta_{j}>0 & \text { for } 1 \leq j \leq N_{\text {free }} \\
\beta_{j}=0 & \text { for } N_{\text {free }}<j \leq N_{\text {slab }}
\end{array}
$$

Then,

$$
\begin{aligned}
\tilde{\mathbf{M}} \mathbf{w}_{j} & =\left(\mathbf{M}-K^{-1} \mathbf{M}_{\text {free }}\right) \mathbf{w}_{j}, \\
& =\mathbf{M} \mathbf{w}_{j}-K^{-1} \beta_{j} \mathbf{M} \mathbf{w}_{j}, \\
& =\left(1-K^{-1} \beta_{j}\right) \mathbf{M} \mathbf{w}_{j},
\end{aligned}
$$

so that the eigenvalues of $\mathbf{M}^{-1} \tilde{\mathbf{M}}$ are of the form $1-K^{-1} \beta_{j}$. As stated above, they are all positive for Fr small enough ( $K$ large). More precisely, they are all positive for $\operatorname{Fr} \leq 1 / \sqrt{\beta_{\text {min }} L_{\text {ship }}}$, where $\beta_{\min }=\min _{j=1}^{N_{\text {free }}} \beta_{j}$. The number of negative eigenvalues increases monotonically with Fr, and for $\mathrm{Fr}>1 / \sqrt{\beta_{\max } L_{\text {ship }}}$ there is a constant number of $N_{\text {free }}$ negative eigenvalues.

We can estimate roughly the value of $\beta_{\text {min }}$ from a simplified 2D analysis. In the $2 \mathrm{D}$ case the slab contains only $N_{\text {free }}=1$ nodes at the free surface and the $\mathbf{M}_{\text {free }}$ and $\mathbf{M}$ matrices are 
of the form (assume constant size elements of length $\Delta z=H / N_{\text {slab }}$ )

$$
\mathbf{M}_{\text {free }}=\left[\begin{array}{cccc}
1 & 0 & \ldots & 0 \\
0 & 0 & \ldots & 0 \\
\vdots & \vdots & \ddots & \vdots \\
0 & 0 & \ldots & 0
\end{array}\right], \quad \mathbf{M}=\Delta z\left[\begin{array}{ccccc}
\frac{1}{3} & \frac{1}{6} & 0 & \ldots & \\
\frac{1}{6} & \frac{2}{3} & \frac{1}{6} & 0 & \ldots \\
& \ddots & \ddots & \ddots & \\
& 0 & \frac{1}{6} & \frac{2}{3} & \frac{1}{6} \\
& & 0 & \frac{1}{6} & \frac{1}{3}
\end{array}\right] \text {. }
$$

The first diagonal element of $\tilde{\mathbf{M}}$ is $\frac{1}{3} \Delta z-K^{-1}$ and changes sign at $K^{-1}=\frac{1}{3} \Delta z$, so that $\beta_{\min } \sim 3 / \Delta z$, and we may estimate that the eigenvalues change sign somewhere near $\operatorname{Fr} \sim\left(\Delta z / 3 L_{\text {ship }}\right)^{\frac{1}{2}}$. Combining these results with that found in Appendix 1, we determine a range of Fr where the FEM calculation applies,

$$
\left(\frac{\Delta z}{3 L_{\text {ship }}}\right)^{\frac{1}{2}} \lesssim \text { Fr } \lesssim\left(\frac{H}{L_{\text {ship }}}\right)^{\frac{1}{2}}
$$

This range is broad enough for applications. The Froude numbers of interest rarely exceed unity, neither do they go below $\mathrm{Fr}=0.1$. As FEM meshes may be be refined exponentially towards the surface, we can get $\Delta z$ small enough at the surface and $H \gg L_{\text {ship }}$, so that restrictions (101) are satisfied.

\section{ACKNOWLEDGMENTS}

This work has received financial support from Consejo Nacional de Investigaciones Científicas y Técnicas (CONICET, Argentina) through Grant BID 802/OC-AR PID No. 26, and from Universidad Nacional del Litoral (Argentina) and was done in collaboration with CIMNE (Centro Internacional de Métodos Numéricos en Ingeniería, Barcelona). We made extensive use of software distributed by the Free Software Foundation/GNU-Project: Linux ELF-OS, Octave from John W. Eaton, Tgif from William C. Cheng, Fortran f2c and g77 compilers, and others. The authors thanks the referees for their constructive suggestions and careful reading.

\section{REFERENCES}

1. C. W. Dawson, A practical computer method for solving ship-wave problems, in Proceedings 2nd International Conference on Numerical Ships Hydrodynamics, Berkeley, 1977, p. 30.

2. J. Broeze and J. E. Romate, Absorbing boundary conditions for free surface wave simulations with a panel method, J. Comput. Phys. 99, 135 (1992).

3. H. C. Raven, A Solution Method for the Nonlinear Ship Wave Resistance Problem, Ph.D. thesis, Technical University Delft, 1996.

4. M. Storti, J. D'Elía, and S. Idelsohn, Computing ship wave resistance from wave amplitude with the DNL absorbing boundary condition, Commun. Numer. Methods Eng., to appear.

5. M. Storti, Implementation details of the absorbing boundary condition for the ship wave-resistance problem. Computing the drag from the wave amplitude, GTM internal report.

6. J. D'Elía, Numerical Methods for the Ship Wave-Resistance Problem, Ph.D. thesis, Universidad Nacional del Litoral, Santa Fe, Argentina, 1997.

7. J. V. Wehausen, The wave resistance of ships, Adv. Appl. Mech. 13, 93 (1973).

8. J. N. Newman, The theory of ship motions, Adv. Appl. Mech. 18, 221 (1978).

9. F. Ogilvie, Singular-perturbation problems in ship hydrodynamics, Adv. Appl. Mech. 17, 92 (1977). 
10. S. Ohring, Three-dimensional ship wave generation using an efficient finite difference scheme with double model linearization, J. Comput. Phys. 41, 89 (1981).

11. Y. Tahara, F. Stern, and B. Rosen, An interactive approach for calculating ship boundary layers and wakes for nonzero Froude number, J. Comput. Phys. 98, 33 (1992).

12. J. Farmer, L. Martinelli, and A. Jameson, Fast multigrid method for solving incompressible hydrodynamic problems with free surface, AIAA J. 32, 1175 (1994).

13. D. Givoli and J. B. Keller, Non-reflecting boundary conditions for elastic waves, Wave Motion 12, 261 (1990).

14. I. Harari and T. J. R. Hughes, Galerkin least-squares finite element methods for the reduced wave equation with non-reflecting boundary conditions in unbounded domains, Comput. Methods Appl. Mech. Eng. 98, 411 (1992).

15. T. Hagstrom and H. B. Keller, Exact boundary conditions at an artificial boundary for partial differential equations in cylinders, SIAM J. Math. Anal. 17, 322 (1986).

16. T. Hagstrom, Boundary conditions at outflow for a problem with transport and diffusion, J. Comput. Phys. 69, 69 (1987).

17. M. Lenoir and A. Tounsi, The localized finite element method and its application to the two-dimensional sea-keeping problem, SIAM J. Numer. Anal. 25, 729 (1988).

18. D. Givoli, Non-reflecting boundary conditions, J. Comput. Phys. 94, 1 (1991).

19. D. Givoli and J. B. Keller, A finite element method for large domains, Comput. Methods Appl. Mech. Eng. 76, 41 (1989).

20. T. Belytschko, Y. Lu, and L. Gu, Element free Galerkin methods, Int. J. Numer. Methods Eng. 37, 229 (1994).

21. E. Oñate, S. Idelsohn, O. C. Zienkiewicz, and T. Fisher, A finite point method for analysis of fluid flow problems, in Proceedings of the 9th International Conference on Finite Element Methods in Fluids, Venice, Italy, October 15-21, 1995.

22. L. Landweber, Motion of immersed and floting bodies, in Handbook of Fluid Dynamics, edited by V. L. Streeter (McGraw-Hill, New York, 1961).

23. B. N. Parlett, The Symmetric Eigenvalue Problem (Prentice-Hall, Englewood Cliffs, NJ, 1980). 